This is an electronic reprint of the original article. This reprint may differ from the original in pagination and typographic detail.

Author(s): Magnani, Valentino; Rajala, Tapio

Title: $\quad$ Radon-Nikodym Property and Area Formula for Banach Homogeneous Group Targets

Year: $\quad 2014$

Version:

Please cite the original version:

Magnani, V., \& Rajala, T. (2014). Radon-Nikodym Property and Area Formula for Banach Homogeneous Group Targets. International Mathematics Research Notices, 2014(23), 6399-6430. https://doi.org/10.1093/imrn/rnt171

All material supplied via JYX is protected by copyright and other intellectual property rights, and duplication or sale of all or part of any of the repository collections is not permitted, except that material may be duplicated by you for your research use or educational purposes in electronic or print form. You must obtain permission for any other use. Electronic or print copies may not be offered, whether for sale or otherwise to anyone who is not an authorised user. 


\title{
RADON-NIKODYM PROPERTY AND AREA FORMULA FOR BANACH HOMOGENEOUS GROUP TARGETS
}

\author{
VALENTINO MAGNANI AND TAPIO RAJALA
}

\begin{abstract}
Aвstract. We prove a Rademacher-type theorem for Lipschitz mappings from a subset of a Carnot group to a Banach homogeneous group, equipped with a suitably weakened Radon-Nikodym property. We provide a metric area formula that applies to these mappings and more generally to all almost everywhere metrically differentiable Lipschitz mappings defined on a Carnot group.
\end{abstract}

\section{Contents}

1. Introduction

2. Banach homogeneous groups

2.1. Two steps Banach homogeneous groups 8

2.2. An infinite product of Heisenberg groups 8

2.3. Infinite products of Engel groups 8

2.4. Banach homogeneous groups that fail the RNP 9

$\begin{array}{ll}\text { 2.5. Infinite products of Carnot groups } & 10\end{array}$

2.6. An infinite product of Lipschitz maps 11

3. Differentiability 12

4. The metric area formula 20

References 23

2010 Mathematics Subject Classification. Primary 58B10, 22E65. Secondary 28A75.

Key words and phrases. Radon-Nikodym property, Rademacher theorem, Carnot group, graded nilpotent Lie group, Banach homogeneous group, metric area formula.

The authors acknowledge the support of the European Project ERC AdG *GeMeThNES*.

The second author also acknowledges the support of the Academy of Finland, project no. 137528. 


\section{INTRODUCTION}

A Banach space $\mathbb{X}$ has the so-called Radon-Nikodym property, in short RNP, when all Lipschitz curves that take values in $\mathbb{X}$ are almost everywhere Fréchet differentiable. Clearly, all $\mathbb{X}$-valued Lipschitz mappings defined on the Euclidean space are almost everywhere Fréchet differentiable if and only if $\mathbb{X}$ has the RNP.

An important space that does not possess the RNP and that plays a role in some questions of theoretical computer science is $L^{1}(0,1)$. In this context, it has been proved in [14] that a new counterexample to the Goemans-Linial conjecture would be a consequence of the nonexistence of bi-Lipschitz embeddings of the first Heisenberg group $\mathbb{H}^{1}$ into $L^{1}(0,1)$. The work [3] by J. Cheeger and B. Kleiner has proved the absence of these embeddings by proving a Rademacher type theorem for these mappings with respect to a suitable notion of differentiability. We consider different classes of Banach spaces that need not have the RNP and we prove that either the Heisenberg group or any other Carnot group, under suitable algebraic conditions, do not admit any bi-Lipschitz embedding into these targets. This will be a consequence of a differentiability result, according to our Theorem 1.1. Subsection 2.4 presents some examples of Banach homogeneous groups that fail to have the RNP and such that our Theorem 1.1 still holds for these targets.

Precisely, our targets are Banach spaces equipped with a Banach Lie group structure, that are metrized by a suitable left invariant distance. In the commutative case, they include classical Banach spaces, but their main feature is that the RNP is only required for a closed and possibly infinite dimensional subspace, that in the finite dimensional framework is known as the horizontal subspace.

These infinite dimensional Lie groups can be naturally called Banach homogeneous groups, since they are a natural extension of their well known finite dimensional version. For the finite dimensional case, the reader can consult for instance [8]. A simple way to present the infinite dimensional versions may consist in requiring the validity of the characterizing properties that hold in the finite dimensional case, as the existence of a group operation with a special structure, the existence of a homogeneous norm, along with dilations and so on. This presentation by axioms can be found in [21].

We follow a different approach, detecting these groups as graded nilpotent Banach Lie algebras, since all the above mentioned properties are just consequences, see Section 2. In fact, one can see a Banach homogeneous group as a Banach space $\mathbb{M}$ equipped with a graded nilpotent Lie product that turns it into a Banach Lie algebra. Thus, we automatically get the group operation by the Dynkin formula for the Baker-Campbell-Hausdorff series, in short $\mathrm{BCH}$, that locally converges in general Banach Lie algebras, [6]. In our case, this series is just a finite sum, since we consider nilpotent Lie algebras.

In sum, we equip $\mathbb{M}$ with three structures, since it is a Banach space, a Banach Lie algebra and also a Banach Lie group. Its main feature is the decomposition into the direct sum $\mathbb{M}=H_{1} \oplus \cdots \oplus H_{\iota}$, where $H_{j}$ are closed subspaces of $\mathbb{M}$, seen as Banach spaces. This yields a precise gradation on the Lie algebra structure of $\mathbb{M}$ that allows us to introduce dilations $\delta_{r}: \mathbb{M} \longrightarrow \mathbb{M}$, that are automatically group isomorphisms. Furthermore, one can also construct a homogeneous norm on $\mathbb{M}$ that respects both the group operation and dilations, hence defining the metric structure of $\mathbb{M}$, see Section 2 for more details. 
As in the finite dimensional case, one can also define the special class of Banach stratified groups, or Carnot groups, since the additional condition that $H_{1}$ Lie generates $\mathbb{M}$ in the finite dimensional case $[8,19]$, can be also stated in the infinite dimensional case. This was already pointed out in the seminal work by M. Gromov, [10]. However, we will focus our attention on the larger class of Banach homogeneous group targets, that presents some additional difficulties in the proof of the almost everywhere differentiability of Lipschitz mappings, as explained below.

Several examples of infinite dimensional Banach homogeneous groups will be presented in Section 2. We mainly exploit a natural product construction by means of the Banach spaces $\ell^{p}$ of $p$-summable sequences. The simplest example of infinite dimensional Banach homogeneous group is the well known Heisenberg group modeled on $H^{2} \times \mathbb{R}$, where $H$ is a real Hilbert space with scalar product $\langle\cdot, \cdot\rangle$. For any $\left(h_{1}, h_{2}, t_{1}\right),\left(h_{1}^{\prime}, h_{2}^{\prime}, t_{2}^{\prime}\right) \in H^{2} \times \mathbb{R}$, the group operation is defined as follows

$$
\left(h_{1}, h_{2}, t_{1}\right)\left(h_{1}^{\prime}, h_{2}^{\prime}, t_{2}^{\prime}\right)=\left(h_{1}+h_{1}^{\prime}, h_{2}+h_{2}^{\prime}, t_{2}+t_{2}^{\prime}+\left\langle h_{1}, h_{2}^{\prime}\right\rangle-\left\langle h_{2}, h_{1}^{\prime}\right\rangle\right) .
$$

This product arises from the quantization relations of the Heisenberg algebra realized in Quantum Mechanics, see for instance Chapter XII, Section 3 of [24]. Notice that this group has an underlying Hilbert space structure. In Subsection 2.2, we introduce the infinite product of Heisenberg groups $\mathbb{H}^{\infty}$, whose underlying Banach structure is given by $\left(\ell^{2}\right)^{2} \times \ell^{1}$. In Subsection 2.5 we present a construction to obtain an infinite product of the same Carnot group. This provides many Banach homogeneous groups whose underlying linear space is a genuinely infinite dimensional Banach space and we will also see that we have some freedom in the choice of the Banach topology. It is clear that one could use a similar construction also for products of different Carnot groups. Motivated by the simple case given by (1) that arises from the Heisenberg group of Quantum Mechanics, one might also expect further physical interpretations for special classes of Banach homogeneous groups.

We wish to clarify that the terminology "Carnot group" will refer throughout to a finite dimensional group. The notion of differentiability between Carnot groups, [19], naturally extends to the case of infinite dimensional Lie group targets, see Definition 3.2. This leads us to the statement of our main result.

Theorem 1.1. Let $\mathbb{M}$ be a Banach homogeneous group such that its subspace $H_{1}$ has the $R N P$. If $\mathbb{G}$ is any Carnot group and $A \subset \mathbb{G}$, then any Lipschitz mapping $f: A \rightarrow \mathbb{M}$ is almost everywhere differentiable.

Since all finite dimensional linear spaces have the RNP, in the special case where $A$ is open and $\mathbb{M}$ is a Carnot group, then Theorem 1.1 also contains the differentiability result by P. Pansu, [19]. If the source space of a Lipschitz mapping is a metric measure space with a Poincaré inequality, namely a PI space, and its target is a Banach space with the RNP, then J. Cheeger and B. Kleiner have established the a.e. differentiability in a suitable sense [2]. However, when the source space is a Carnot group, the differentiability from a PI space does not imply the differentiability in the sense of Carnot groups, where "non-rectifiable directions" are also considered, see Definition 3.2. Nevertheless, differentiability on PI spaces can still provide bi-Lipschitz non-embedding theorems into Banach spaces with the RNP, although this differentiability does not include the case of Lie group targets. It 
is also easy to observe that a special case of Theorem 1.1 implies the bi-Lipschitz nonembeddability of noncommutative Carnot groups into any Banach space with the RNP. In fact, these Banach spaces clearly constitute the simplest case of commutative Banach homogeneous groups, whose first layer has the RNP.

In connection with area formulae and change of variables in Carnot groups, subsequent works have extended this Rademacher type theorem to the case where $A$ is any subset of a Carnot group $\mathbb{G}$ and $\mathbb{M}$ is another Carnot group, [16], [22]. Further differentiability results in this vein are also written for targets corresponding to equiregular Carnot-Carathéodory spaces, see [12] and references therein. On the other hand, in all of these works a key point is that all targets are also length metric spaces and this allows one to use the existence of one dimensional Lipschitz extensions in order to achieve the a.e. differentiability on an arbitrary subset. In Theorem 1.1 the target need not be a length metric space, therefore this theorem turns out to be new also when the codomain is a finite dimensional homogeneous group that is not stratified. In fact, we overcome this difficulty by Theorem 3.1, that is the key tool. Here we establish the almost everywhere differentiability of Lipschitz curves defined on an arbitrary subset of the real line and that take values in an arbitrary Banach homogeneous group $\mathbb{M}$. Although this Lipschitz curve need not have a Lipschitz extension with respect to the homogeneous distance $\rho$ on $\mathbb{M}$, we use its Lipschitz extension with respect to the Banach norm. This leads us to the a.e. differentiability of the projection of the curve into the first layer of the target, that has the RNP. Then we use the geometric properties of the density points, along with a suitable application of the Dynkin formula, exploiting the explicit expression for the addends appearing in the finite expansion of the group operation, [5]. This provides a new and simpler approach to the a.e. differentiability of graded group-valued Lipschitz mappings defined on an arbitrary subset of the real line.

A natural issue related to Theorem 1.1 concerns the existence of nontrivial Lipschitz mappings. We wish to make sure that there are Lipschitz mappings that are not a mere composition of a Carnot group-valued Lipschitz mapping with a Lipschitz embedding into a Banach homogeneous group. In Subsection 2.6, we construct Lipschitz mappings that cannot have the form previously described. In fact, we consider a suitable infinite product of a family of Lipschitz mappings $\left\{f^{k}\right\}_{k \geq 0}$, under the condition that all vanish at some point. The corresponding product mapping $G$ turns out to be a Lipschitz mapping taking values in the infinite product $\mathbb{H}^{\infty}$ introduced in Subsection 2.2. In the case all mappings $f^{k}$ do not vanish at some point, the corresponding product mapping $G$ is an example of Lipschitz mapping with infinite dimensional image. Since the horizontal layer of $\mathbb{H}^{\infty}$ has the RNP, our Theorem 1.1 shows that $G$ is also almost everywhere differentiable, when the source space is any Carnot group.

A stronger condition than the nonexistence of bi-Lipschitz embeddings is that of pure $\mathbb{G}$ unrectifiability. A purely $\mathbb{G}$-unrectifiable metric space $(Y, \rho)$ has the property that the image of any $Y$-valued Lipschitz mapping from a subset of the $Q$-dimensional Carnot group $\mathbb{G}$ has vanishing Hausdorff measure $\mathcal{H}_{\rho}^{Q}$. In particular, this implies that there does not exist any bi-Lipschitz embedding of $\mathbb{G}$ into the metric space $Y$. In the finite dimensional setting, the area formula leads to an algebraic characterization of purely $\mathbb{G}$-unrectifiable stratified groups, along with rigidity theorems, [17]. The simplest instance of this characterization is that any set of positive measure in the Heisenberg group (or any noncommutative Carnot 
group) does not bi-Lipschitz embed into any fixed Euclidean space, as was first observed by S. Semmes, [23]. This naturally introduces the question of a suitable area formula from a measurable set of a Carnot group into an infinite dimensional target. We will present a rather general area formula that also includes the mappings of Theorem 1.1.

For a general metric space target, the choice of the source space is crucial. In fact, for metric space-valued Lipschitz mappings on a subset of a Euclidean space, B. Kirchheim has proved their almost everywhere metric differentiability and also the corresponding area formula, [13]. Carnot groups have a sufficiently rich structure to introduce the notion of metric differentiability, when any of their subsets constitutes the source space, see Definition 4.1. In this case, the metric differential is given by a homogeneous seminorm, namely a continuous function $s: \mathbb{G} \longrightarrow\left[0,+\infty\left[\right.\right.$ such that $s(x)=s\left(x^{-1}\right), s\left(\delta_{r} x\right)=r s(x)$ and $s(x \cdot y) \leq s(x)+s(y)$ for all $x, y \in \mathbb{G}$ and $r>0$. The additional condition that $s(x)=0$ implies $x=0$ means that $s$ is a homogeneous norm.

On one side, when the source space is a noncommutative Carnot group, such as the Heisenberg group, then counterexamples to the metric differentiability of Lipschitz mappings can be constructed, [15]. On the other side, if we restrict metric differentiability to horizontal directions, then we still have an almost everywhere (horizontal) metric differentiation for metric space-valued Lipschitz mappings on Carnot groups, [20].

Theorem 1.1 clearly provides nontrivial cases where metric space-valued Lipschitz mappings on Carnot groups are almost everywhere metrically differentiable. Other new targets where the almost everywhere metric differentiability holds can be found by another recent result of J. Cheeger and B. Kleiner, [4]. In fact, one can notice that the seminorm $\|\cdot\|_{x}$ of Theorem 1.3 in [4] can be seen as a homogeneous seminorm on the whole Heisenberg group $\mathbb{H}$, therefore the limit in the statement of this theorem, with $z_{2}$ equal to the unit element, exactly yields the almost everywhere metric differentiability of Lipschitz mappings from $\mathbb{H}$ to $L^{1}(0,1)$, see also [3]. We observe that in all previously mentioned cases, where the almost everywhere metric differentiability holds, one can apply the following new metric area formula.

Theorem 1.2. Let $A \subset \mathbb{G}$ be measurable, let $f: A \longrightarrow Y$ be Lipschitz and almost everywhere metrically differentiable. It follows that

$$
\int_{A} J(m d f(x)) d \mathcal{H}_{d}^{Q}(x)=\int_{Y} N(f, y) d \mathcal{H}_{\rho}^{Q}(y),
$$

where $N(f, y)=\sharp\left(f^{-1}(y)\right)$ for all $y \in Y$ is the multiplicity function, $d$ is the homogeneous distance of $\mathbb{G}, \rho$ is the metric of $Y$ and $Q$ is the Hausdorff dimension of $\mathbb{G}$.

As usual, the point of an area formula is its notion of Jacobian. The metric Jacobian $J(s)$ of the homogeneous seminorm $s$ is defined as follows

$$
J(s)=\left\{\begin{array}{ll}
\frac{\mathcal{H}_{s}^{Q}\left(B_{1}\right)}{\mathcal{H}_{d}^{Q}\left(B_{1}\right)} & \text { if } s \text { is a homogeneous norm } \\
0 & \text { otherwise }
\end{array} .\right.
$$

If $\mathbb{G}$ is a Euclidean space, then (3) yields the Jacobian of [13]. If the target is a Banach homogeneous group $\mathbb{M}$ equipped with a distance $\rho$ given by a homogeneous norm, then 
we have to observe that differentiability with differential $L: \mathbb{G} \longrightarrow \mathbb{M}$ implies metric differentiability with homogeneous seminorm $h \rightarrow s_{L}(h)=\rho(L(h), 0)$ with $h \in \mathbb{G}$. Thus, we get a more explicit formula for (3), that in the special case where $\mathbf{M}$ is another Carnot group fits into the sub-Riemannian Jacobian introduced in [16], see Remark 4.1 for more comments.

Concerning the proof of (2), a substantial difference in our approach with respect to that of [13] is in the proof of the negligibility of the image of points where the metric differential is not a homogeneous norm. In [13], this fact is achieved by combining the integral representation of Kirchheim's Jacobian with the use of $\varepsilon$-approximating graph extensions of the mapping, as in [7]. Our argument is surprisingly more elementary, since it only uses the very definition of metric differentiability without any use of the notion of metric Jacobian, see Lemma 4.3.

By the metric area formula (2), for each Carnot group $\mathbb{G}$, a Banach homogeneous group whose horizontal subspace has the RNP is purely $\mathbb{G}$-unrectifiable if none of its homogeneous subgroups is h-isomorphic to $\mathbb{G}$, see Definition 3.1. In particular, a Banach space with the RNP is purely $\mathbb{G}$-unrectifiable whenever $\mathbb{G}$ is noncommutative. If $\mathbb{G}$ has step higher than two, then any two step Banach homogeneous group whose horizontal subspace has the RNP must be purely $\mathbb{G}$-unrectifiable and so on. We have already mentioned that whenever a metric space $Y$ is purely $\mathbb{G}$-unrectifiable, then in particular it cannot admit any bi-Lipschitz embedding from $\mathbb{G}$ into $Y$. As a consequence, the previous theorems on purely $\mathbb{G}$-unrectifiability of some Banach spaces automatically provide new bi-Lipschitz non-embeddability theorems into infinite dimensional targets.

\section{BANACH HOMOGENEOUS GROUPS}

We start from the notion of Banach Lie algebra, namely a Banach space $\mathbb{M}$ equipped with a continuous, bilinear and skew-symmetric mapping $[\cdot, \cdot]: \mathbb{M} \times \mathbb{M} \longrightarrow \mathbb{M}$ that satisfies the Jacobi identity. A nilpotent Banach Lie algebra $\mathbb{M}$ is characterized by the existence of a positive integer $v \in \mathbb{N}$ such that whenever $x_{1}, x_{2}, \ldots, x_{v+1} \in \mathbb{M}$, we have

$$
\left.\left.\left[\cdots\left[\left[x_{1}, x_{2}\right], x_{3}\right] \cdots\right], x_{v}\right], x_{v+1}\right]=0
$$

and there exist $y_{1}, y_{2}, \ldots, y_{v} \in \mathbb{M}$ such that

$$
\left.\left[\cdots\left[\left[y_{1}, y_{2}\right], y_{3}\right] \cdots\right], y_{v}\right] \neq 0 .
$$

The integer $v$ is uniquely defined and it gives the step of nilpotence of $\mathbb{M}$. Therefore the algebra $\mathbb{M}$ can be equipped with a canonical Banach Lie group operation

$$
x y=x+y+\sum_{m=2}^{v} P_{m}(x, y),
$$

that is the "truncated" Baker-Campbell-Hausdorff series. For any $m \geq 2$, the polynomial $P_{m}$ is given by the Dynkin's formula

$$
P_{m}(x, y)=\sum \frac{(-1)^{k-1}}{k} \frac{m^{-1}}{p_{1} ! q_{1} ! \cdots p_{k} ! q_{k} !} \underbrace{x \circ \cdots \circ x}_{p_{1} \text { times }} \circ \overbrace{y \circ \cdots \circ y}^{q_{1} \text { times }} \cdots \circ \underbrace{x \circ \cdots \circ x}_{p_{k} \text { times }} \circ \overbrace{y \circ \cdots \circ y}^{q_{k} \text { times }},
$$


where $\left.x_{i_{1}} \circ x_{i_{2}} \circ \cdots \circ x_{i_{k}}=\left[\cdots\left[\left[x_{i_{1}}, x_{i_{2}}\right], x_{i_{3}}\right] \cdots\right], x_{i_{k}}\right]$ and the sum is taken over the $2 k$-tuples $\left(p_{1}, q_{1}, p_{2}, q_{2}, \ldots, p_{k}, q_{k}\right)$ such that $p_{i}+q_{i} \geq 1$ for all positive $i, k \in \mathbb{N}$ and $\sum_{i=1}^{k} p_{i}+q_{i}=m$. Notice that $P_{2}(x, y)=[x, y] / 2$. Formula (5) was established by E. B. Dynkin in [5]. We say that $\mathbb{M}$ equipped with the group operation (4) is a Banach nilpotent Lie group. If we denote by $L(\mathbb{M})$ the Lie algebra of $\mathbb{M}$ as a Lie group, we may wonder whether $L(\mathbb{M})$ is isomorphic to $\mathbb{M}$ seen as a Lie algebra equipped with the initially given Lie product $[\cdot, \cdot]$. The answer to this question is yes, according to the following proposition, whose proof can be established by the use of the $\mathrm{BCH}$ series for the group expansion.

Proposition 2.1. If $\mathbb{M}$ is a Banach nilpotent Lie group, then the given Lie algebra structure on $\mathbb{M}$ is isomorphic to $L(\mathbb{M})$.

If $S_{1}, S_{2}, \ldots, S_{n} \subset \mathbb{X}$ are closed subspaces of a Banach space $\mathbb{X}$ such that the mapping $J: S_{1} \times \cdots \times S_{n} \longrightarrow \mathbb{X}$ with $J\left(s_{1}, \ldots, s_{n}\right)=\sum_{l=1}^{n} s_{l}$ is an isomorphism of Banach spaces, then $\mathbb{X}=S_{1} \oplus \cdots \oplus S_{n}$ denotes the corresponding direct sum. Any canonical projection on $S_{j}$ is denoted by $\pi_{j}: \mathbb{X} \longrightarrow S_{j}$.

Definition 2.1. We say that the Banach space $\mathbb{M}$ is a Banach homogeneous group if it is equipped with a Banach Lie product $[\cdot, \cdot]: \mathbb{M} \times \mathbb{M} \longrightarrow \mathbb{M}$ and there exist $\iota$ closed subspaces $H_{1}, \ldots, H_{\iota}$ such that $\mathbb{M}=H_{1} \oplus \cdots \oplus H_{\iota}$ and whenever $x \in H_{i}$ and $y \in H_{j}$ we have $[x, y] \in H_{i+j}$ if $i+j \leq \iota$ and $[x, y]=0$ otherwise. This equips $\mathbb{M}$ with a special family of Banach isomorphisms $\delta_{r}: \mathbb{M} \longrightarrow \mathbb{M}, r>0$, defined by $\delta_{r} x=r^{i} x$ if $x \in H_{i}$ for all $i=1, \ldots, \iota$. These mappings are both group and algebra automorphisms of $\mathbb{M}$ and are called dilations.

Remark 2.1. A Banach homogeneous group can be seen as a Banach graded nilpotent Lie group equipped with dilations. This is the natural terminology from the finite dimensional case of graded Lie groups, see $[8,9]$. The decomposition $\mathbb{M}=H_{1} \oplus \cdots \oplus H_{\iota}$ with the properties stated in the previous definition defines a gradation of $\mathbb{M}$.

The gradation of $\mathbb{M}$ along with the Dynkin formula (5) yields some positive constants $\sigma_{1}, \ldots, \sigma_{\iota}$, depending on the norm of the Lie product, such that

$$
\|x\|=\max \left\{\sigma_{i}\left|x_{i}\right|^{1 / i}: 1 \leq i \leq \iota\right\}
$$

with $\sigma_{1}=1$, satisfies $\left\|\delta_{r} x\right\|=r\|x\|$ and $\|x y\| \leq\|x\|+\|y\|$. We have denoted by $|\cdot|$ the underlying norm on $\mathbb{M}$ that makes it a Banach space. This convention will be understood in the sequel. The properties of $\|\cdot\|$ that we have previously seen, allow us to say that $\|\cdot\|$ is a Banach homogeneous norm of $\mathbb{M}$.

If we set $\rho(x, y)=\left\|x^{-1} y\right\|$, then we have obtained a left invariant homogeneous distance on $\mathbb{M}$ with respect to the group operation such that $\rho\left(\delta_{r} x, \delta_{r} y\right)=r \rho(x, y)$ for all $x, y \in \mathbb{M}$ and $r>0$. We say that $\rho$ is a Banach homogeneous distance on $\mathbb{M}$. In the sequel, we assume that every Banach homogeneous group $\mathbb{M}$ is equipped with the Banach homogeneous norm (6) and the corresponding homogeneous distance $\rho$, unless otherwise stated.

For the subsequent examples, we recall the standard class of Banach spaces

$$
\ell^{p}=\left\{\left(x_{k}\right)_{k \geq 0} \in \mathbb{R}^{\mathbb{N}}: \sum_{k=0}^{\infty}\left|x_{k}\right|^{p}<\infty\right\}
$$


where $p \geq 1$ is any real number and $\left|\left(x_{k}\right)_{k \geq 0}\right|_{p}=\left(\sum_{k=0}^{\infty}\left|x_{k}\right|^{p}\right)^{1 / p}$.

2.1. Two steps Banach homogeneous groups. It is not difficult to construct the general model for a two step Banach homogeneous group. We consider two Banach spaces $\mathbb{X}$ and $\mathbb{T}$. We have the Banach space $\mathbb{G}_{2}=\mathbb{X} \oplus \mathbb{T}$ with the product norm. The structure of Banach homogeneous group is given by the bounded skew-symmetric bilinear form $\beta: \mathbb{X} \times \mathbb{X} \rightarrow \mathbb{T}$ via the formula $\left[(x, t),\left(x^{\prime}, t^{\prime}\right)\right]=\left(0, \beta\left(x, x^{\prime}\right)\right)$ for all $x, x^{\prime} \in \mathbb{X}$ and $t, t^{\prime} \in \mathbb{T}$. Thus, the Lie group operation on $\mathbb{G}_{2}$ is given by the following formula

$$
(x, t) \cdot\left(x^{\prime}, t^{\prime}\right)=\left(x+x^{\prime}, t+t^{\prime}\right)+\left[(x, t),\left(x^{\prime}, t^{\prime}\right)\right]=\left(x+x^{\prime}, t+t^{\prime}+\beta\left(x, x^{\prime}\right)\right) .
$$

Let $|(x, t)|=|x|_{\mathbf{X}}+|y|_{\mathbb{T}}$ denote the product norm in the Banach space $\mathbb{G}_{2}$. Let $c>0$ be such that $\left|\beta\left(x, x^{\prime}\right)\right|_{\mathbb{T}} \leq c|x|_{\mathbb{X}}\left|x^{\prime}\right|_{\mathbf{X}}$ for all $x, x^{\prime} \in \mathbb{X}$ and fix any constant $\sigma>0$ such that $\sigma \leq \sqrt{2 / c}$. Then the function $\|(x, t)\|=\max \left\{|x|_{\mathbb{X}}, \sigma|t|_{\mathbb{T}}^{1 / 2}\right\}$ defines a homogeneous norm on $\mathbb{G}_{2}$ and clearly for any $r>0$ the group isomorphism $\delta_{r}(x, t)=\left(r x, r^{2} t\right)$ for $(x, t) \in \mathbb{G}_{2}$ is a dilation of $\mathbb{G}_{2}$. In sum, only the mapping $\beta$ suffices to equip $\mathbb{G}_{2}$ with the structure of two step Banach homogeneous group.

2.2. An infinite product of Heisenberg groups. We wish to consider a concrete example of nontrivial two step Banach homogeneous group. This group, that we denote by $\mathbb{H}^{\infty}$, can be seen as a suitably topologized infinite product of the same Heisenberg group. As a Banach space $\mathbb{H}^{\infty}$ coincides with $\left(\ell^{2}\right)^{2} \times \ell^{1}$, where the horizontal subspace is $\mathbb{X}=\left(\ell^{2}\right)^{2}$ and $\mathbb{T}=\ell^{1}$. Any element $x \in \mathbb{H}^{\infty}$ corresponds to $\left(x_{1}, x_{2}, x_{3}\right)$ where $x_{i}=\left(x_{i j}\right)_{j \geq 0}$. We also write $\left|x_{i}\right|_{2}=\sqrt{\sum_{j=0}^{\infty} x_{i j}^{2}}$ for $i=1,2$ and $\left|x_{3}\right|_{1}=\sum_{j=0}^{\infty}\left|x_{3 j}\right|$. For any $x, y \in \mathbb{H}^{\infty}$, we define the skew-symmetric bilinear mapping $\beta:\left(\ell^{2}\right)^{2} \times\left(\ell^{2}\right)^{2} \longrightarrow \ell^{1}$ as follows

$$
\beta\left(\left(x_{1}, x_{2}\right),\left(y_{1}, y_{2}\right)\right)=\left(0,0,\left(x_{1 j} y_{2 j}-x_{2 j} y_{1 j}\right)_{j \geq 0}\right) .
$$

It follows that for all $\left(x_{1}, x_{2}\right),\left(y_{1}, y_{2}\right) \in\left(\ell^{2}\right)^{2}$ we have

$$
\left|\beta\left(\left(x_{1}, x_{2}\right),\left(y_{1}, y_{2}\right)\right)\right|_{1} \leq\left(\left|x_{1}\right|_{2}^{2}+\left|x_{2}\right|_{2}^{2}\right)^{1 / 2}\left(\left|y_{1}\right|_{2}^{2}+\left|y_{2}\right|_{2}^{2}\right)^{1 / 2} \text {. }
$$

According to the general model of two step Banach homogeneous group, the function $\left\|\left(x_{1}, x_{2}, x_{3}\right)\right\|=\max \left\{\sqrt{\left|x_{1}\right|_{2}^{2}+\left|x_{2}\right|_{2}^{2}}, \sqrt{\left|x_{3}\right|_{1}}\right\}$ defines a homogeneous norm on $\mathbb{H}^{\infty}$.

2.3. Infinite products of Engel groups. Let us consider the Engel group $\mathbb{E}$ with graded decomposition $S_{1} \oplus S_{2} \oplus S_{3}$ and graded basis $\left(e_{11}, e_{12}, e_{3}, e_{4}\right)$, namely $\left(e_{11}, e_{12}\right),\left(e_{3}\right)$ and $\left(e_{4}\right)$ are bases of $S_{1}, S_{2}$ and $S_{3}$, respectively. The only nontrivial bracket relations of $\mathbb{E}$ as a Lie algebra are $\mathcal{L}\left(e_{11}, e_{12}\right)=e_{3}$ and $\mathcal{L}\left(e_{11}, e_{3}\right)=e_{4}$, then $\mathcal{L}: \mathbb{E} \times \mathbb{E} \longrightarrow \mathbb{E}$ defines a Lie product on $\mathbb{E}$. We define $H_{1}=\left(\ell^{2}\right)^{2}, H_{2}=\ell^{p}, H_{3}=\ell^{q}$ and set $\mathbb{E}^{\infty}=H_{1} \times H_{2} \times H_{3}$, where $1 \leq p \leq 2$ and $q \geq 1$. An element $x$ of $\mathbb{E}^{\infty}$ can be written as $\left(x_{1}, x_{2}, x_{3}\right)$, where $x_{1}=\left(x_{11}, x_{12}\right), x_{1 i}=\left(x_{1 i}^{k}\right)_{k \geq 0} \in \ell^{2}, i=1,2, x_{2}=\left(x_{2}^{k}\right)_{k \geq 0} \in \ell^{1}$ and $x_{3}=\left(x_{3}^{k}\right)_{k \geq 0} \in \ell^{1}$.

We observe that for all $\xi, \eta \in \ell^{2}$ and $\zeta \in \ell^{p}$, we have

$$
\left|\left(\xi^{k} \eta^{k}\right)_{k \geq 0}\right|_{p} \leq \sum_{k=0}^{\infty}\left|\xi^{k} \eta^{k}\right| \leq|\xi|_{2}|\eta|_{2} \quad \text { and } \quad\left|\left(\xi^{k} \zeta^{k}\right)_{k \geq 0}\right|_{q} \leq \sum_{k=0}^{\infty}\left|\xi^{k} \zeta^{k}\right| \leq|\xi|_{2}|\eta|_{2} \leq|\xi|_{2}|\eta|_{p} .
$$


Thus, the products $\xi \cdot \eta=\sum_{k=0}^{\infty} \xi^{k} \eta^{k} e_{p}^{k} \in \ell^{p}$ and $\xi \cdot \zeta=\sum_{k=0}^{\infty} \xi^{k} \zeta^{k} e_{q}^{k} \in \ell^{q}$ are well defined, where $\left(e_{p}^{k}\right)_{k \geq 0}$ and $\left(e_{q}^{k}\right)_{k \geq 0}$ are the canonical Schauder basis of $\ell^{p}$ and $\ell^{q}$, respectively. A nice point in the construction of $\mathbb{E}^{\infty}$ is that we do not need to construct the group operation, but it suffices to construct a continuous Lie product. Thus, we set

$$
[x, y]=\left(0, x_{11} \cdot y_{12}-x_{12} \cdot y_{11}, x_{11} \cdot y_{2}-x_{2} \cdot y_{11}\right) .
$$

We fix the product Banach norm $|x|=\left|x_{11}\right|_{2}+\left|x_{12}\right|_{2}+\left|x_{2}\right|_{p}+\left|x_{3}\right|_{q}$ and observe that

$$
|[x, y]| \leq 4|x||y|
$$

showing the continuity of $[\cdot, \cdot]$ with respect to the Banach norm $|\cdot|$. The Jacobi identity follows from the one of $\mathcal{L}(\cdot, \cdot)$.

Remark 2.2. Notice that the arbitrary choice of $p \in[1,2]$ and $q \in[1,+\infty)$ in the infinite product of Engel groups emphasizes, as one could expect, that there are infinitely many Banach topologies that we can use for our construction of a Banach homogeneous group. This fact will be also seen in Subsection 2.5.

2.4. Banach homogeneous groups that fail the RNP. It is possible to construct Banach homogeneous groups that do not have the RNP, although their first layer has this property. As a consequence, these targets satisfy the assumptions of Theorem 1.1.

A simple example is a Banach Heisenberg group $\mathbb{H}=H_{1} \oplus H_{2}$, where $H_{1}=L^{2}(0,1)^{2}$ and $H_{2}=L^{1}(0,1)$. We define $\beta: H_{1} \times H_{1} \rightarrow H_{2}$ as

$$
\beta\left(\left(u_{1}, v_{1}\right),\left(u_{2}, v_{2}\right)\right)=u_{1} v_{2}-u_{2} v_{1}
$$

that clearly satisfies $\left\|\beta\left(\left(u_{1}, v_{1}\right),\left(u_{2}, v_{2}\right)\right)\right\|_{L^{1}(0,1)} \leq\left\|\left(u_{1}, v_{1}\right)\right\|_{L^{2}(0,1)^{2}}\left\|\left(u_{2}, v_{2}\right)\right\|_{L^{2}(0,1)^{2}}$. Using the group operation of Subsection 2.1, we have defined a two step homogeneous group that fails the RNP and whose first layer has the RNP.

Let us now construct a Banach Engel group that fails to have the RNP. We define the Banach space $\mathbb{E}=H_{1} \oplus H_{2} \oplus H_{3}$, where

$$
H_{1}=L^{3}(0,1) \times L^{3}(0,1), \quad H_{2}=L^{3 / 2}(0,1), \quad \text { and } \quad H_{3}=L^{1}(0,1) .
$$

For $i=1,2$, we consider $x_{i} \in \mathbb{E}$ as $\left(u_{i}, v_{i}, z_{i}, t_{i}\right)$, where $u_{i}, v_{i} \in L^{3}(0,1), z_{i} \in L^{3 / 2}(0,1)$ and $t_{i} \in L^{1}(0,1)$. Then define Lie product

$$
\left[x_{1}, x_{2}\right]=\left(0,0, u_{1} v_{2}-u_{2} v_{1}, u_{1} z_{2}-u_{2} z_{1}\right) .
$$

We fix the Banach norm $\left|x_{i}\right|=\left|u_{i}\right|_{L^{3}(0,1)}+\left|v_{i}\right|_{L^{3}(0,1)}+\left|z_{i}\right|_{L^{3 / 2}(0,1)}+\left|t_{i}\right|_{L^{1}(0,1)}$ and observe that

$$
\left|\left[x_{1}, x_{2}\right]\right| \leq 4\left|x_{1}\right|\left|x_{2}\right| \text {. }
$$

It follows that the Lie product $[\cdot, \cdot]$ is continuous with respect to $|\cdot|$. The Jacobi identity is a simple verification. We also notice that the first layer of $\mathbb{E}$ has the RNP, hence Theorem 1.1 also applies to this target. 
2.5. Infinite products of Carnot groups. The previous cases suggest a general "product construction" for any graded group $\mathbb{G}=S_{1} \oplus \cdots \oplus S_{v}$. Thus, we set $\mathbb{G}^{\infty}=H_{1} \times \cdots \times H_{v}$, where $H_{i}=\left(\ell^{p_{i}}\right)^{n_{i}}$ and $n_{i}=\operatorname{dim} S_{i}$ for all $i=1, \ldots, v$ and the real numbers $p_{i} \geq 1$, whenever $1 \leq i, j \leq v$ and $i+j \leq v$, satisfy the following inequality

$$
p_{i+j} \geq \frac{1}{2} \max \left\{p_{i}, p_{j}\right\}
$$

For any $i=1, \ldots, v$ we set the basis $\left(e_{i 1}, \ldots, e_{i n_{i}}\right)$ of $S_{i}$, hence $\left(e_{i u}\right)_{\substack{1 \leq i \leq n \\ 1 \leq u \leq n_{i}}}$ is a basis of $\mathbb{G}$. For an element $x$ of $\mathbb{G}^{\infty}$ we will use the equivalent notation $\left(x_{1}, \ldots, x_{v}\right)$, where $x_{i}=\left(x_{i 1}, \ldots, x_{i n_{i}}\right)$ and $x_{i u}=\left(x_{i u}^{k}\right)_{k \geq 0} \in \ell^{p_{i}}$. We set the norms

$$
\left|x_{i}\right|_{p_{i}}=\left(\sum_{1 \leq u \leq n_{i}} \sum_{k \geq 0}\left|x_{i u}^{k}\right|^{p_{i}}\right)^{1 / p_{i}} \text { and }|x|=\sum_{i=1}^{v}\left|x_{i}\right|_{p_{i}} .
$$

Notice that we can also write $\left|x_{i}\right|_{p_{i}}=\left(\sum_{1 \leq u \leq n_{i}}\left(\left|x_{i u}\right|_{p_{i}}\right)^{p_{i}}\right)^{1 / p_{i}}$. Using the previous notation, for any $x \in \mathbb{G}^{\infty}$ we set

$$
x^{k}=\sum_{\substack{1 \leq i \leq v \\ 1 \leq u \leq n_{i}}} x_{i j}^{k} e_{i u} \in \mathbb{G} .
$$

We denote by $\mathcal{L}$ the Lie product of $\mathbb{G}$, then for any $x, y \in \mathbb{G}^{\infty}$ and any $k \in \mathbb{N}$, we set

$$
\mathcal{L}\left(x^{k}, y^{k}\right)=\sum_{\substack{1 \leq i \leq v \\ 1 \leq u \leq n_{i}}} \mathcal{L}_{i u}\left(x^{k}, y^{k}\right) e_{i u} \in \mathbb{G} .
$$

We also define $\mathcal{L}^{k}(x, y)=\mathcal{L}\left(x^{k}, y^{k}\right)$ and $\mathcal{L}_{i u}^{k}(x, y)=\mathcal{L}_{i u}\left(x^{k}, y^{k}\right)$. Taking into account that whenever $1 \leq i, j \leq v, i+j \leq v, 1 \leq u \leq n_{i}, 1 \leq v \leq n_{j}$, we have

$$
\mathcal{L}\left(e_{i u}, e_{j v}\right)=\sum_{1 \leq r \leq n_{i+j}} \beta_{i u, j v}^{r} e_{(i+j) r}
$$

for some coefficients $\beta_{i u, j v}^{r}$ that determine the Lie algebra structure of $\mathbb{G}$, we expand $\mathcal{L}\left(x^{k}, y^{k}\right)$ using (9), establishing the formula

$$
\mathcal{L}_{i r}^{k}(x, y)=\sum_{\substack{1 \leq a, b \leq v, a+b=i, 1 \leq u \leq n, 1 \leq v \leq n_{b}}} \beta_{a u, b v}^{r} x_{a u}^{k} y_{b v}^{k}
$$

where $k \in \mathbb{N}, i=2, \ldots, v$ and $r=1, \ldots, n_{i}$. As a consequence, we introduce the elements

$$
\mathcal{L}_{i r}(x, y)=\left(\mathcal{L}_{i r}^{k}(x, y)\right)_{k \geq 0} \quad \text { and } \quad \mathcal{L}_{i}(x, y)=\left(\mathcal{L}_{i 1}(x, y), \ldots, \mathcal{L}_{i n_{i}}(x, y)\right) .
$$

By elementary computations, one can check that there exist constants $C_{1 i}>0$ such that

$$
\left|\mathcal{L}_{i r}^{k}(x, y)\right|^{p_{i}} \leq C_{1 i} \sum_{\substack{1 \leq a, b \leq v \\ a+b=i}}\left(\left|x_{a}^{k}\right|_{p_{a}}\right)^{p_{i}}\left(\left|y_{b}^{k}\right|_{p_{b}}\right)^{p_{i}}
$$

where $x_{a}^{k}=\left(x_{a 1}^{k}, \ldots, x_{a n_{a}}^{k}\right)$ and $\left(\left|x_{a}^{k}\right|_{p_{a}}\right)^{p_{a}}=\sum_{r=1}^{n_{a}}\left|x_{a r}^{k}\right|^{p_{a}}$ for any $x \in \mathbb{G}^{\infty}$ and any $a=1, \ldots, v$. Thus, we can consider the sum with respect to $k$ and $r$, getting constants $C_{2 i}>0$ such that

$$
\sum_{1 \leq r \leq n_{i}} \sum_{k=0}^{\infty}\left|\mathcal{L}_{i r}^{k}(x, y)\right|^{p_{i}} \leq C_{2 i} \sum_{\substack{1 \leq a, b \leq v \\ a+b=i}} \sum_{k=0}^{\infty}\left(\left|x_{a}^{k}\right|_{p_{a}}\right)^{p_{i}}\left(\left|y_{b}^{k}\right|_{p_{b}}\right)^{p_{i}} \text {. }
$$


Finally, we observe that

$$
\left(\sum_{k=0}^{\infty}\left(\left|x_{a}^{k}\right|_{p_{a}}\right)^{p_{i}}\left(\left|y_{b}^{k}\right|_{p_{b}}\right)^{p_{i}}\right)^{1 / p_{i}} \leq\left|\left(\left|x_{a}^{k}\right|_{p_{a}}\right)_{k \geq 0}\right|_{2 p_{i}}\left|\left(\left|y_{b}^{k}\right|_{p_{b}}\right)_{k \geq 0}\right|_{2 p_{i}}
$$

and the condition (7) yields

$$
\sum_{k=0}^{\infty}\left(\left|x_{a}^{k}\right|_{p_{a}}\right)^{p_{i}}\left(\left|y_{b}^{k}\right|_{p_{b}}\right)^{p_{i}} \leq\left(\left|\left(\left|x_{a}^{k}\right|_{p_{a}}\right)_{k \geq 0}\right|_{p_{a}}\left|\left(\left|y_{b}^{k}\right|_{p_{b}}\right)_{k \geq 0}\right|_{p_{b}}\right)^{p_{i}} .
$$

Taking into account (8), we have $\left|\left(\left|x_{a}^{k}\right|_{p_{a}}\right)_{k \geq 0}\right|_{p_{a}}=\left|x_{a}\right|_{p_{a}}$ and $\left|\left(\left|y_{b}^{k}\right|_{p_{b}}\right)_{k \geq 0}\right|_{p_{b}}=\left|y_{b}\right|_{p_{b}}$. As a result, taking into account (10), we get

$$
\left(\left|\mathcal{L}_{i}(x, y)\right|_{p_{i}}\right)^{p_{i}}=\sum_{1 \leq r \leq n_{i}} \sum_{k=0}^{\infty}\left|\mathcal{L}_{i r}^{k}(x, y)\right|^{p_{i}} \leq C_{2 i} \sum_{\substack{1 \leq a, b \leq v \\ a+b=i}}\left(\left|x_{a}\right|_{p_{a}}\right)^{p_{i}}\left(\left|y_{b}\right|_{p_{b}}\right)^{p_{i}} \leq C_{2 i} v^{2}|x|^{p_{i}}|y|^{p_{i}},
$$

that immediately implies that

$$
[x, y]=\left(0, \mathcal{L}_{2}(x, y), \ldots, \mathcal{L}_{v}(x, y)\right) \in \mathbb{G}^{\infty} \quad \text { and } \quad|[x, y]| \leq \sum_{i=2}^{v}\left(C_{2_{i}} v^{2}\right)^{1 / p_{i}}|x||y| .
$$

Finally, the Jacobi identity for the product $[\cdot, \cdot]$ follows from the Jacobi identity of $\mathcal{L}$.

Remark 2.3. It is clear that the previous "product construction" can be suitably generalized to the cases of different Carnot groups. The obvious case is taking the product of $\mathbb{G}^{\infty}$ with a different Carnot group $\mathbb{G}_{1}$, but many other similar possibilities can arise.

2.6. An infinite product of Lipschitz maps. Let us consider any sequence of Lipschitz mappings $f^{k}: X \longrightarrow \mathbb{H}$, where $(X, d)$ is a metric space and $\mathbb{H}$ is the first Heisenberg group equipped with the homogeneous norm $\left|\left(\xi_{1}, \xi_{2}, \xi_{3}\right)\right|_{\mathbb{H}}=\max \left\{\left|\left(\xi_{1}, \xi_{2}\right)\right|, \sqrt{\left|\xi_{3}\right|}\right\}$ and the group operation $\left(\xi_{1}, \xi_{2}, \xi_{3}\right)\left(\eta_{1}, \eta_{2}, \eta_{3}\right)=\left(\xi_{1}+\eta_{1}, \xi_{2}+\eta_{2}, \xi_{3}+\eta_{3}+\xi_{1} \eta_{2}-\xi_{2} \eta_{1}\right)$. We have denoted by $|\cdot|$ both the Euclidean norm in $\mathbb{R}^{2}$ and in $\mathbb{R}$. Up to left translations, we can assume that for some $x_{0} \in X$ we have

$$
f^{k}\left(x_{0}\right)=0 \quad \text { for all } \quad k \in \mathbb{N} \text {. }
$$

Let us define $\operatorname{Lip}\left(f^{k}\right)=\sup _{x, y \in X, x \neq y}\left\{\left|f^{k}(x)^{-1} f^{k}(y)\right|_{\mathbb{H}} / d(x, y)\right\}$, then we set $L_{k}=\operatorname{Lip}\left(f^{k}\right)$ and select any sequence $\left(r_{k}\right)_{k \geq 0}$ of positive numbers such that

$$
C_{0}=\left(\sum_{k=0}^{\infty} r_{k}^{2} L_{k}^{2}\right)^{1 / 2}<+\infty
$$

We wish to construct the infinite product of the mappings $g_{k}=\delta_{r_{k}} \circ f^{k}$, where $k \in \mathbb{N}$. We expect that the new target is the infinite product $\mathbb{H}^{\infty}=\left(\ell^{2}\right)^{2} \times \ell^{1}$, defined in Subsection 2.2. Following the notations of this subsection, we set $f^{k}(x)=\left(f_{11}^{k}(x), f_{12}^{k}(x), f_{2}^{k}(x)\right) \in \mathbb{H}$, so that

$$
g_{k}(x)=\left(r_{k} f_{11}^{k}(x), r_{k} f_{12}^{k}(x), r_{k}^{2} f_{2}^{k}(x)\right) \in \mathbb{H} \quad \text { for all } k \in \mathbb{N} .
$$

Setting $g^{k}(x)=\left(g_{11}^{k}(x), g_{12}^{k}(x), g_{2}^{k}(x)\right) \in \mathbb{H}$, we define $G_{1 j}(x)=\left(g_{1 j}^{k}(x)\right)_{k \geq 0}$ with $j=1,2$ and $G_{2}(x)=\left(g_{2}^{k}(x)\right)_{k \geq 0}$. Clearly, $\operatorname{Lip}\left(g^{k}\right)=r_{k} L_{k}$, therefore condition (11) yields

$$
\max \left\{\left|g_{1}^{k}(x)\right|, \sqrt{\left|g_{2}^{k}(x)\right|}\right\}=\left|g^{k}(x)\right|_{\mathbb{H}} \leq r_{k} L_{k} d\left(x, x_{0}\right)
$$


where $g_{1}^{k}(x)=\left(g_{11}^{k}(x), g_{12}^{k}(x)\right)$. By (12), it follows that $G_{11}(x), G_{12}(x) \in \ell^{2}$ and $G_{2}(x) \in \ell^{1}$. As a consequence, we have that $\left(G_{1}(x), G_{2}(x)\right) \in \mathbb{H}^{\infty}$ for all $x \in X$, where we have defined $G_{1}(x)=\left(G_{11}(x), G_{12}(x)\right)$. We use both the norm $\|\cdot\|$ and the group operation introduced in Subsection 2.2 for the Banach homogeneous group $\mathbb{H}^{\infty}$. With these notions, for the mapping $G: X \longrightarrow \mathbb{H}^{\infty}$ defined as $G(x)=\left(G_{1}(x), G_{2}(x)\right)$ for $x \in X$, we have

$\left\|G(x)^{-1} G(y)\right\|=\max \left\{\left|-G_{1}(x)+G_{1}(y)\right|_{2}, \sqrt{\left|-G_{2}(x)+G_{2}(y)-G_{11}(x) \cdot G_{12}(y)+G_{12}(x) \cdot G_{11}(x)\right|_{1}}\right\}$

where we have used the product $z \cdot w=\sum_{k=0}^{\infty} z_{j} w_{j} e_{j} \in \ell^{1}$, where $z, w \in \ell^{2}$ and $\left(e_{k}\right)_{k \geq 0}$ is the canonical Schauder basis of $\ell^{1}$. The condition (12) finally leads us to the following Lipschitz continuity

$$
\left\|G(x)^{-1} G(y)\right\| \leq C_{0} d(x, y) \text { for all } \quad x, y \in X .
$$

\section{DifFERENTIABILITY}

This section is devoted to the proof of Theorem 1.1. We equip a Carnot group $\mathbb{G}$ with a continuous left invariant distance $d$ such that $d\left(\delta_{r} x, \delta_{r} y\right)=r d(x, y)$ for all $x, y \in \mathbb{G}$ and $r>0$, namely, a homogeneous distance. The set $B_{x, r} \subset \mathbb{G}$ denotes the open ball of center $x$ and radius $r$ with respect to $d$. When the center $x$ of the open ball is the origin, namely the unit element of $\mathbb{G}$, we simply write $B_{r}$. The same rule is used for closed balls $D_{x, r}$ of center $x$ and radius $r>0$. The set of density points $D(A)$ of $A \subset \mathbb{G}$ is formed by all $x \in \mathbb{G}$ with

$$
\lim _{r \rightarrow 0^{+}} \frac{\mathcal{H}_{d}^{Q}\left(A \cap B_{x, r}\right)}{\mathcal{H}_{d}^{Q}\left(B_{x, r}\right)}=1 .
$$

In the sequel, $\mathbb{M}$ is a Banach homogeneous group with gradation $H_{1} \oplus \cdots \oplus H_{\iota}$ and equipped with homogeneous norm $\|\cdot\|$ given by (6). The Carnot group $\mathbb{G}$ has the decomposition into the direct sum $S_{1} \oplus \cdots \oplus S_{v}$, where the layers satisfy the condition $\left[S_{1}, S_{j}\right]=S_{j+1}$ for all $j=1, \ldots, v-1$ and $\left[S_{1}, S_{v}\right]=\{0\}$.

Definition 3.1. A homogeneous homomorphism, in short h-homomorphism, from $\mathbb{G}$ to $\mathbb{M}$ is a continuous Lie group homomorphism $L: \mathbb{G} \longrightarrow \mathbb{M}$ such that $L\left(\delta_{r}^{\mathbb{G}} x\right)=\delta_{r}^{\mathbb{M}} L(x)$ for all $x \in \mathbb{G}$ and $r>0$, where $\delta_{r}^{\mathbb{G}}$ and $\delta_{r}^{\mathbb{M}}$ are dilations in $\mathbb{G}$ and $\mathbb{M}$, respectively.

Definition 3.2. Let $A \subset \mathbb{G}$ and let $\mathbb{M}$ be a Banach homogeneous group equipped with a Banach homogeneous distance $\rho$. We say that $f: A \longrightarrow \mathbb{M}$ is differentiable at the density point $x \in A$ if there exists an h-homomorphism $L: \mathbb{G} \longrightarrow \mathbb{M}$ such that

$$
\rho\left(f(x)^{-1} f(x z), L(z)\right)=o(d(z, 0))
$$

as $z \in x^{-1} A$ and $d(z, 0) \rightarrow 0^{+}$. The mapping $L$ is the differential of $f$ at $x$, that is uniquely defined and denoted by $D f(x)$.

In the sequel, saying that $H_{1}$ has the $R N P$ precisely means that the restriction of the Banach norm of $\mathbb{M}$ onto $H_{1}$ turns this closed subspace into a Banach space with the RNP.

Theorem 3.1. Let $\mathbb{M}$ be a Banach graded Lie group such that $H_{1}$ has the RNP. Let $A \subset \mathbb{R}$ and let $\gamma: A \rightarrow \mathbb{M}$ be a Lipschitz mapping. Then $\gamma$ is almost everywhere differentiable. 
Proof. We can obviously assume that $A$ is closed, since the target is a complete metric space. Our Lipschitz bound on $\left\|\delta_{\frac{1}{h}}\left(\gamma(t)^{-1} \gamma(t+h)\right)\right\|$ for all $t, t+h \in A$ implies that

$$
\frac{\left|\gamma_{j}(t+h)-\gamma_{j}(t)+\sum_{m=2}^{\iota} P_{m j}(-\gamma(t), \gamma(t+h))\right|}{h^{j}}
$$

is also bounded, where $P_{m j}=\pi_{j} \circ P_{m}$. It is not restrictive to assume that $h>0$ and $A$ is bounded. Since $\mathbb{M}$ is a Banach space and $\gamma$ is also Lipschitz with respect to the Banach norm, we can apply the [11], to get Lipschitz extension $f$ defined on a bounded interval containing $A$. In the sequel, we denote by $f_{j}$ the mapping $\pi_{j} \circ f$ for all $j+1, \ldots, \iota$. The Radon-Nikodym property of $H_{1}$ implies that $f_{1}$ is a.e. differentiable on the bounded interval containing $A$. For a.e. $t$ in the bounded interval, we have

$$
\frac{1}{h} \int_{t-h}^{t+h}\left|\dot{f}_{1}(s)-\dot{f}_{1}(t)\right| d s \rightarrow 0 \quad \text { as } \quad h \rightarrow 0^{+},
$$

where the integral is understood to be the Bochner integral, see [1, Chapter 5] for the basic properties of the Bochner integral. We can restrict our attention to all density points $t$ of $A$ that are also points of differentiability and such that $\dot{f}_{1}$ satisfies (13). Now, we fix any point $t$ of $A$ having these properties.

We consider the left translated $g(s)=f(t)^{-1} f(s) \in \mathbb{M}$. We also set $g_{j}=\pi_{j} \circ g$ for all $j=1, \ldots, \iota$, observing that $g_{1}(s)=f_{1}(s)-f_{1}(t) \in H_{1}$. Both mappings $g$ and $g_{1}$ are Lipschitz continuous with respect to $d$ and $|\cdot|$, respectively, and have the same Lipschitz constants of $f$ and $f_{1}$, respectively.

If we fix $n \in \mathbb{N} \backslash\{0\}$, then we have $h_{n, t}>0$, depending on $n$ and $t$, such that for all $h \in(A-t) \cap] 0, h_{n, t}\left[\right.$ we have $\operatorname{dist}\left(A, t+\frac{i}{n} h\right)<\frac{i}{n^{2}} h \leq \frac{h}{n}$ for $i=0, \ldots, n$. Thus, there exist points $t_{i} \in A$, for $i=0,1, \ldots, n$, so that

$$
\left|t+\frac{i}{n} h-t_{i}\right| \leq \frac{h}{n}
$$

$t_{0}=t$ and $t_{n}=t+h$. We write $g_{j}(t+h)=A_{j}-B_{j}$, where

$$
\left\{\begin{array}{l}
A_{j}=\sum_{i=0}^{n-1}\left(g_{j}\left(t_{i+1}\right)-g_{j}\left(t_{i}\right)+\sum_{m=2}^{\iota} P_{m j}\left(-g\left(t_{i}\right), g\left(t_{i+1}\right)\right)\right) \\
B_{j}=\sum_{i=0}^{n-1} \sum_{m=2}^{\iota} P_{m j}\left(-g\left(t_{i}\right), g\left(t_{i+1}\right)\right)
\end{array} .\right.
$$

We observe that

$$
\left|A_{j}\right| \leq \sum_{i=0}^{n-1}\left|\pi_{j}\left(g\left(t_{i}\right)^{-1} g\left(t_{i+1}\right)\right)\right|=\sum_{i=0}^{n-1}\left|\pi_{j}\left(f\left(t_{i}\right)^{-1} f\left(t_{i+1}\right)\right)\right|,
$$

hence $A_{j}$ is bounded by $\sum_{i=0}^{n-1}\left|t_{i+1}-t_{i}\right|^{j}$, up to a constant factor only depending on the Lipschitz constant of $f$. Since $\left|t_{i+1}-t_{i}\right| \leq 3 h / n$, we get a constant $\kappa_{1 j}>0$ such that

$$
\left|A_{j}\right| \leq \kappa_{1 j} h^{j} / n^{j-1} .
$$

We denote by $l_{0}>0$ a number only depending on the Lipschitz constant of $f$ and such that

$$
\max \left\{\left\|f\left(t^{\prime}\right)^{-1} f\left(t^{\prime \prime}\right)\right\|,\left|f\left(t^{\prime}\right)-f\left(t^{\prime \prime}\right)\right|,\left|f_{1}\left(t^{\prime}\right)-f_{1}\left(t^{\prime \prime}\right)\right|, \ldots,\left|f_{\iota}\left(t^{\prime}\right)-f_{\iota}\left(t^{\prime \prime}\right)\right|\right\} \leq l_{0}\left|t^{\prime}-t^{\prime \prime}\right|
$$


for all $t^{\prime}, t^{\prime \prime}$ belonging to the bounded interval containing $A$. By the Dynkin formula (5), the term $P_{m j}\left(-g\left(t_{i}\right), g\left(t_{i+1}\right)\right)$ in (15) can be written as a linear combination of

$$
\pi_{j}\left(\Delta_{i} \circ g\left(\tau_{1, i}\right) \circ \cdots \circ g\left(\tau_{m-2, i}\right)\right),
$$

where $\Delta_{i} \in\left\{-g\left(t_{i}\right) \circ g\left(t_{i+1}\right), g\left(t_{i+1}\right) \circ\left(-g\left(t_{i}\right)\right)\right\}$ and $\tau_{1, i}, \ldots, \tau_{m-2, i} \in\left\{t_{i}, t_{i+1}\right\}$. Therefore we have $\Delta_{i} \in\left\{ \pm g\left(t_{i}\right) \circ g\left(t_{i+1}\right)\right\}$ and the previous term can be written as

$$
\pm \pi_{j}\left(g\left(t_{i}\right) \circ\left(g\left(t_{i+1}\right)-g\left(t_{i}\right)\right) \circ g\left(\tau_{1, i}\right) \circ \cdots \circ g\left(\tau_{m-2, i}\right)\right) .
$$

Up to a change of sign, this term is the sum of elements

$$
\left(g_{l_{1}}\left(t_{i+1}\right)-g_{l_{1}}\left(t_{i}\right)\right) \circ g_{l_{2}}\left(t_{i}\right) \circ g_{l_{3}}\left(\tau_{1, i}\right) \circ \cdots \circ g_{l_{m}}\left(\tau_{m-2, i}\right),
$$

where $1 \leq l_{1}, \ldots, l_{m}<j$ and $l_{1}+\cdots+l_{m}=j$. Recall that the range of $m \in \mathbb{N}$ is $2 \leq m \leq j$. We start with the case $m=j$, where the element to consider is

$$
\left(g_{1}\left(t_{i+1}\right)-g_{1}\left(t_{i}\right)\right) \circ g_{1}\left(t_{i}\right) \circ g_{1}\left(\tau_{1, i}\right) \circ \cdots \circ g_{1}\left(\tau_{m-2, i}\right) .
$$

We can write this element as follows

$$
\left(\int_{t_{i}}^{t_{i+1}} \dot{g}_{1}\right) \circ\left[\left(\int_{t}^{t_{i}} \dot{g}_{1}-\dot{g}_{1}(t)\right)+\left(t_{i}-t\right) \dot{g}_{1}(t)\right] \circ g_{1}\left(\tau_{1, i}\right) \cdots \circ g_{1}\left(\tau_{m-2, i}\right) .
$$

This can be considered as the sum of $\left(\int_{t_{i}}^{t_{i+1}} \dot{g}_{1}\right) \circ\left(\int_{t}^{t_{i}} \dot{g}_{1}-\dot{g}_{1}(t)\right) \circ g_{1}\left(\tau_{1, i}\right) \circ \cdots \circ g_{1}\left(\tau_{m-2, i}\right)$ and $\left(\int_{t_{i}}^{t_{i+1}} \dot{g}_{1}\right) \circ\left(\left(t_{i}-t\right) \dot{g}_{1}(t)\right) \circ g_{1}\left(\tau_{1, i}\right) \circ \cdots \circ g_{1}\left(\tau_{m-2, i}\right)$. The norm of the first element is not larger than $l_{0}^{j-2} h^{j-2}\left(\int_{\min \left\{t_{i}, t_{i+1}\right\}}^{\max \left\{t_{i}, t_{i+1}\right\}}\left|\dot{g}_{1}\right|\right)\left(\int_{t}^{t+h}\left|\dot{g}_{1}-\dot{g}_{1}(t)\right|\right)$, hence we get

(21) $\left|\left(\int_{t_{i}}^{t_{i+1}} \dot{g}_{1}\right) \circ\left(\int_{t}^{t_{i}} \dot{g}_{1}-\dot{g}_{1}(t)\right) \circ \cdots \circ g_{1}\left(\tau_{m-2, i}\right)\right| \leq l_{0}^{j-2} h^{j-2}\left(\int_{t+\frac{(i-1)}{n} h}^{t+\frac{(i+2)}{n} h}\left|\dot{g}_{1}\right|\right)\left(\int_{t}^{t+h}\left|\dot{g}_{1}-\dot{g}_{1}(t)\right|\right)$.

The second one can be written as

$$
\left(\int_{t_{i}}^{t_{i+1}} \dot{g}_{1}-\dot{g}_{1}(t)\right) \circ\left(\left(t_{i}-t\right) \dot{g}_{1}(t)\right) \circ g_{1}\left(\tau_{1, i}\right) \circ \cdots \circ g_{1}\left(\tau_{m-2, i}\right)
$$

hence its norm is less than or equal to $l_{0}^{j-1} h^{j-1} \int_{t+\frac{(i-1)}{n} h}^{t+\frac{(i+2)}{n} h}\left|\dot{g}_{1}-\dot{g}_{1}(t)\right|$. We have proved that $\left|g_{1}\left(t_{i+1}\right) \circ g_{1}\left(t_{i}\right) \circ g_{1}\left(\tau_{1, i}\right) \circ \cdots \circ g_{1}\left(\tau_{m-2, i}\right)\right|$ is less than

$$
l_{0}^{j-2} h^{j-2}\left(\int_{t+\frac{(i-1)}{n} h}^{t+\frac{(i+2)}{n} h}\left|\dot{g}_{1}\right|\right)\left(\int_{t}^{t+h}\left|\dot{g}_{1}-\dot{g}_{1}(t)\right|\right)+l_{0}^{j-1} h^{j-1} \int_{t+\frac{(i-1)}{n} h}^{t+\frac{(i+2)}{n} h}\left|\dot{g}_{1}-\dot{g}_{1}(t)\right| .
$$

This proves that

(22) $\left|g_{1}\left(t_{i+1}\right) \circ g_{1}\left(t_{i}\right) \circ \cdots \circ g_{1}\left(\tau_{m-2, i}\right)\right| \leq 3 l_{0}^{j-1} h^{j-1}\left(\frac{1}{n} \int_{t}^{t+h}\left|\dot{g}_{1}-\dot{g}_{1}(t)\right|+\int_{t+\frac{(i-1)}{n} h}^{t+\frac{(i+2)}{n} h}\left|\dot{g}_{1}-\dot{g}_{1}(t)\right|\right)$. 
Notice that in the case $j=2$, the estimate (22) can be read as

$$
\left|\sum_{m=2}^{2} P_{m 2}\left(-g_{1}\left(t_{i}\right), g_{1}\left(t_{i+1}\right)\right)\right| \leq \frac{3}{2} l_{0} h\left(\frac{1}{n} \int_{t}^{t+h}\left|\dot{g}_{1}-\dot{g}_{1}(t)\right|+\int_{t+\frac{(i-1)}{n} h}^{t+\frac{(i+2)}{h} h}\left|\dot{g}_{1}-\dot{g}_{1}(t)\right|\right),
$$

for all $0<h<h_{n, t}, t+h \in A$ and all possible choices of $t_{i} \in A$ satisfying (14), that clearly also depend on $h$. Arguing by induction, suppose that

$$
\left|\sum_{m=2}^{l} P_{m l}\left(-g\left(t_{i}\right), g\left(t_{i+1}\right)\right)\right| \leq \kappa_{2 l} h^{l-1}\left(\frac{h}{n^{2}}+\frac{1}{n} \int_{t-h}^{t+h}\left|\dot{g}_{1}-\dot{g}_{1}(t)\right|+\int_{t+\frac{(i-1)}{n} h}^{t+\frac{(i+2)}{n} h}\left|\dot{g}_{1}-\dot{g}_{1}(t)\right|\right)
$$

holds for all $l=2, \ldots, j$, for all $t, t+h \in A, 0<h<h_{n, t, j}$ and any choice of $t_{i} \in A$ satisfying (14) for all $i=1, \ldots, n-1, t_{0}=t$ and $t_{n}=t+h$. In view of (23), the induction hypothesis is true for $j=2$. Thus, for any $t \in A$, we have to find an $h_{n, t, j+1}>0$ such that

$$
\left|\sum_{m=2}^{j+1} P_{m(j+1)}\left(-g\left(t_{i}\right), g\left(t_{i+1}\right)\right)\right| \leq \kappa_{2(j+1)} h^{j}\left(\frac{h}{n^{2}}+\frac{1}{n} \int_{t-h}^{t+h}\left|\dot{g}_{1}-\dot{g}_{1}(t)\right|+\int_{t+\frac{(i-1)}{n} h}^{t+\frac{(i+2)}{n} h}\left|\dot{g}_{1}-\dot{g}_{1}(t)\right|\right)
$$

for any choice of $t_{i} \in A$ satisfying (14) and all $0<h<h_{n, t, j+1}$ such that $t+h \in A$. Since $g_{l}(t+h)=A_{l}-B_{l}$, we observe that our inductive assumption yields

$$
\left|g_{l}(t+h)\right| \leq 3 \kappa_{2 l} h^{l-1} \int_{t-h}^{t+h}\left|\dot{g}_{1}-\dot{g}_{1}(t)\right|+\left(\kappa_{1 l}+\kappa_{2 l}\right) \frac{h^{l}}{n}
$$

for any $t, t+h \in A$ with $0<h<h_{n, t, j}$ and all $l=2, \ldots, j$, where $j \geq 2$. Arguing as in the previous steps, by the Dynkin formula, the single addends $P_{m(j+1)}\left(-g\left(t_{i}\right), g\left(t_{i+1}\right)\right)$ with $2 \leq m \leq j$ are finite sums of elements

$$
\left(g_{l_{1}}\left(t_{i+1}\right)-g_{l_{1}}\left(t_{i}\right)\right) \circ g_{l_{2}}\left(t_{i}\right) \circ g_{l_{3}}\left(\tau_{1, i}\right) \circ \cdots \circ g_{l_{m}}\left(\tau_{m-2, i}\right),
$$

where $1 \leq l_{1}, \ldots, l_{m} \leq j, l_{1}+\cdots+l_{m}=j+1$ and $2 \leq m \leq j+1$. If $m=j+1$, then the general validity of (22) in our case gives

(28) $\left|g_{1}\left(t_{i+1}\right) \circ g_{1}\left(t_{i}\right) \circ \cdots \circ g_{1}\left(\tau_{m-2, i}\right)\right| \leq 3 l_{0}^{j} h^{j}\left(\frac{1}{n} \int_{t-h}^{t+h}\left|\dot{g}_{1}-\dot{g}_{1}(t)\right|+\int_{t+\frac{(i-1)}{n} h}^{t+\frac{(i+2)}{n} h}\left|\dot{g}_{1}-\dot{g}_{1}(t)\right|\right)$

for all $0<h<h_{n, t}$ and all $t_{i} \in A$ satisfying (14). Let us now consider the case $2 \leq m<j+1$, where we have to apply our inductive hypothesis. Concerning (27) we have two main cases. The first one is when $l_{1}=1$. Thus, we consider the set

$$
J_{0}=\left\{j \in \mathbb{N}: 2 \leq j \leq m, l_{j} \geq 2\right\} .
$$

Since $m<j+1$, we have $J_{0} \neq \emptyset$. Precisely, $J_{0}$ is made by $p$ distinct elements $\left\{j_{1}, \ldots, j_{p}\right\}$ with $1 \leq p \leq m-1$. We also set $I_{0}=\{2, \ldots, m\} \backslash J_{0}$. If $p<m-1$, namely $I_{0} \neq \emptyset$, then $I_{0}=\left\{i_{1}, \ldots, i_{m-p-1}\right\}$ and $l_{i}=1$ for all $i \in I_{0}$. By the consequence (26) of the inductive assumption, since $0<t_{i+1}-t, t_{i}-t \leq h<h_{n, t, j}$, it follows that the norm of (27) is less than or equal to

$$
l_{0}\left|t_{i+1}-t_{i}\right|\left(l_{0} h\right)^{m-p-1} \prod_{l=l_{j_{1}}, \ldots, l_{j_{p}}}\left(3 \kappa_{2 l} h^{l-1} \int_{t-h}^{t+h}\left|\dot{g}_{1}-\dot{g}_{1}(t)\right|+\left(\kappa_{1 l}+\kappa_{2 l}\right) \frac{h^{l}}{n}\right)
$$


for all $0<h<h_{t, n, j}$, with $t+h \in A$. Thus, there exists $\kappa_{3}(m, p)>0$, only depending on $l_{0}$, $\kappa_{1 l}$ and $\kappa_{2 l}$, for all $l=2, \ldots, j$, such that

$$
\left|\left(g_{l_{1}}\left(t_{i+1}\right)-g_{l_{1}}\left(t_{i}\right)\right) \circ g_{l_{2}}\left(t_{i}\right) \circ g_{l_{3}}\left(\tau_{1, i}\right) \circ \cdots \circ g_{l_{m}}\left(\tau_{m-2, i}\right)\right| \leq \kappa_{3}(m, p) h^{j}\left(\frac{1}{n} \int_{t-h}^{t+h}\left|\dot{g}_{1}-\dot{g}_{1}(t)\right|+\frac{h}{n^{2}}\right) .
$$

Explicitly, we can choose $\kappa_{3}(m, p)=9 l_{0}^{m-p}\left(12 l_{0}+1\right)^{p-1}\left(\max _{2 \leq l \leq j} \kappa_{1 l}+\kappa_{2 l}\right)^{p}$.

The remaining case is $l_{1} \geq 2$. We observe that for the integers $l$ such that $2 \leq l \leq \iota$, the consequence (26) of the inductive assumption yields

$$
\left|g_{l}\left(t^{\prime}\right)\right| \leq\left(t^{\prime}-t\right)^{l}\left(\kappa_{1 l}+\kappa_{2 l}+12 \kappa_{2 l} l_{0}\right)
$$

for all $t^{\prime} \in A$ such that $0<t^{\prime}-t<h_{n, t, j}$. In the case $l=1$, we have $\left|g_{1}\left(t^{\prime}\right)\right| \leq l_{0}\left(t^{\prime}-t\right)$ for all $t^{\prime} \in A \cap(t,+\infty)$. Now, the general term (27) can be written as the sum of

$$
\left(g_{l_{1}}\left(t_{i+1}\right)-g_{l_{1}}\left(t_{i}\right)+\sum_{m=2}^{l_{1}} P_{m l_{1}}\left(-g\left(t_{i}\right), g\left(t_{i+1}\right)\right)\right) \circ g_{l_{2}}\left(t_{i}\right) \circ g_{l_{3}}\left(\tau_{1, i}\right) \circ \cdots \circ g_{l_{m}}\left(\tau_{m-2, i}\right)
$$

and of

$$
\left(\sum_{m=2}^{l_{1}} P_{m l_{1}}\left(-g\left(t_{i}\right), g\left(t_{i+1}\right)\right)\right) \circ g_{l_{2}}\left(t_{i}\right) \circ g_{l_{3}}\left(\tau_{1, i}\right) \circ \cdots \circ g_{l_{m}}\left(\tau_{m-2, i}\right) .
$$

Since the first factor of (31) is $\pi_{l_{1}}\left(g\left(t_{i}\right)^{-1} g\left(t_{i+1}\right)\right)$, the norm of (31) is not greater than

$$
l_{0}^{l_{1}} \frac{\left|t_{i}-t_{i+1}\right|^{l_{1}}}{\sigma_{l_{1}}}\left(\kappa_{1 l}+\kappa_{2 l}+l_{0}+12 \kappa_{2 l} l_{0}\right)^{m-1} h^{l_{2}+\cdots+l_{m}} .
$$

Now, we observe that the norm of (32) is less than or equal to the following number

$$
\left|\sum_{m=2}^{l_{1}} P_{m l_{1}}\left(-g\left(t_{i}\right), g\left(t_{i+1}\right)\right)\right|\left|g_{l_{2}}\left(t_{i}\right)\right|\left|g_{l_{3}}\left(\tau_{1, i}\right)\right| \cdots\left|g_{l_{m}}\left(\tau_{m-2, i}\right)\right| .
$$

By the induction hypothesis (24), the first factor of this product is less than or equal to

$$
\kappa_{2 l_{1}} h^{l_{1}-1}\left(\frac{h}{n^{2}}+\frac{1}{n} \int_{t-h}^{t+h}\left|\dot{g}_{1}-\dot{g}_{1}(t)\right|+\int_{t+\frac{(i-1)}{n} h}^{t+\frac{(i+2)}{n} h}\left|\dot{g}_{1}-\dot{g}_{1}(t)\right|\right),
$$

hence, as before, taking into account (26), the product (34) is not larger than

$$
\kappa_{2 l_{1}} h^{l_{1}-1}\left(\frac{h}{n^{2}}+\frac{1}{n} \int_{t-h}^{t+h}\left|\dot{g}_{1}-\dot{g}_{1}(t)\right|+\int_{t+\frac{(i-1)}{n} h}^{t+\frac{(i+2)}{n} h}\left|\dot{g}_{1}-\dot{g}_{1}(t)\right|\right) \kappa_{4}(m) h^{l_{2}+\cdots+l_{m}},
$$

where $\kappa_{4}(m)=\left(\kappa_{1 l}+\kappa_{2 l}+l_{0}+12 \kappa_{2 l} l_{0}\right)^{m-1}$. Taking into account the decomposition of (27) into the sum of (31) and (32) and using the estimates (33) and (35), we get a geometric constant $\kappa_{5}(m)$, only depending on $l_{0}$ and all $\kappa_{1, l}$ and $\kappa_{2 l}$ with $l=2, \ldots, j$, such that

$$
\frac{\left|\left(g_{l_{1}}\left(t_{i+1}\right)-g_{l_{1}}\left(t_{i}\right)\right) \circ g_{l_{2}}\left(t_{i}\right) \circ \cdots \circ g_{l_{m}}\left(\tau_{m-2, i}\right)\right|}{\kappa_{5}(m) h^{j}} \leq \frac{h}{n^{2}}+\frac{1}{n} \int_{t-h}^{t+h}\left|\dot{g}_{1}-\dot{g}_{1}(t)\right|+\int_{t+\frac{(i-1)}{n} h}^{t+\frac{(i+2)}{n} h}\left|\dot{g}_{1}-\dot{g}_{1}(t)\right|
$$


in the case $l_{1} \geq 2$. Joining both cases $l_{1}=1$ and $l_{1} \geq 2$, namely, joining (29) with (36), we get a new constant $\kappa_{6}(m) \geq \kappa_{5}(m)$ depending on the same constants of $\kappa_{5}(m)$, such that

$$
\frac{\left|\left(g_{l_{1}}\left(t_{i+1}\right)-g_{l_{1}}\left(t_{i}\right)\right) \circ g_{l_{2}}\left(t_{i}\right) \circ \cdots \circ g_{l_{m}}\left(\tau_{m-2, i}\right)\right|}{\kappa_{6}(m) h^{j}} \leq \frac{h}{n^{2}}+\frac{1}{n} \int_{t-h}^{t+h}\left|\dot{g}_{1}-\dot{g}_{1}(t)\right|+\int_{t+\frac{(i-1)}{n} h}^{t+\frac{(i+2)}{n} h}\left|\dot{g}_{1}-\dot{g}_{1}(t)\right|
$$

whenever $2 \leq m \leq j+1, l_{1}, l_{2}, \ldots, l_{m} \geq 1, l_{1}+l_{2}+\cdots+l_{m}=j+1, t, t+h \in A$, $0<h<\min \left\{h_{n, t, j}, h_{n, t}\right\}$ and $t_{i} \in A$ satisfy (14) for all $i=1, \ldots, n-1$, where $t_{0}=t$ and $t_{n}=t+h$. Thus, under the same conditions, since $P_{m(j+1)}\left(-g\left(t_{i}\right), g\left(t_{i+1}\right)\right)$ is a finite linear combination of elements (27), we also have

$$
\left|P_{m(j+1)}\left(-g\left(t_{i}\right), g\left(t_{i+1}\right)\right)\right| \leq \kappa_{7}(m) h^{j}\left(\frac{h}{n^{2}}+\frac{1}{n} \int_{t-h}^{t+h}\left|\dot{g}_{1}-\dot{g}_{1}(t)\right|+\int_{t+\frac{(i-1)}{n} h}^{t+\frac{(i+2)}{n} h}\left|\dot{g}_{1}-\dot{g}_{1}(t)\right|\right)
$$

for a suitable constant $\kappa_{7}(m)>0$, depending on $\kappa_{6}(m)$. This immediately leads us to $(25)$ and concludes our argument by induction. As a consequence, taking into account that $g_{j}(t+h)=A_{j}-B_{j}$ and $B_{j}=\sum_{i=0}^{n-1} \sum_{m=2}^{j} P_{m j}\left(-g\left(t_{i}\right), g\left(t_{i+1}\right)\right)$, we obtain

$$
\left|g_{j}(t+h)\right| \leq 3 \kappa_{2 j} h^{j-1} \int_{t-2 h}^{t+2 h}\left|\dot{g}_{1}-\dot{g}_{1}(t)\right|+\left(\kappa_{1 j}+\kappa_{2 j}\right) \frac{h^{j}}{n}
$$

for all $j=2, \ldots, \iota$ and all $0<h<h_{n, t, j}$. It follows that

$$
\limsup _{h \rightarrow 0} \frac{\left|g_{j}(t+h)\right|}{|h|^{j}} \leq\left(\kappa_{1 j}+\kappa_{2 j}\right) \frac{1}{n},
$$

hence for the arbitrary choice of $n \in \mathbb{N} \backslash\{0\}$, we have proved that there exists

$$
\lim _{\substack{h \rightarrow 0 \\ t+h \in A}} \delta_{\frac{1}{h}}\left(\gamma(t)^{-1} \gamma(t+h)\right) \text {. }
$$

This concludes the proof.

Proof of Theorem 1.1. We can clearly assume that $A$ is closed, since $f$ is Lipschitz and the target is a complete metric space. It is also not restrictive to assume that $A$ is also bounded. Let $v_{1}, v_{2}, \ldots, v_{N} \in S_{1}$ be a set of horizontal directions of $\mathbb{G}$ such that $\left|v_{i}\right|=1$ for all $i=1, \ldots, N$ and for some $T>0$ we have that

$$
V=\left\{\delta_{t_{1}} v_{1} \cdots \delta_{t_{N}} v_{N}:\left|t_{i}\right|<T\right\}
$$

is a neighbourhood of the origin containing $B_{1}$. For all $x \in \mathbb{G}$ and $v \in \mathbb{G} \backslash\{0\}$ we write the one dimensional set of parameters for which we hit the set $A$ as $A(x, v)=\left\{t \in \mathbb{R} \mid x \delta_{t} v \in A\right\}$. Take one of the directions $v_{i}$ and denote by $Z_{i}$ the one-dimensional subgroup spanned by it. Let $W_{i}$ be the complementary subgroup, so that $\mathbb{G}$ is the semidirect product of $Z_{i}$ and $W_{i}$. Then by Theorem 3.1 for any $x \in W_{i}$ the limit

$$
\lim _{\substack{t \rightarrow 0 \\ t \in A\left(x \delta_{s} v_{i}, v_{i}\right)}} \delta_{\frac{1}{t}}\left(f\left(x \delta_{s} v_{i}\right)^{-1} f\left(x \delta_{s} v_{i} \delta_{t} v_{i}\right)\right)
$$

exists for $\mathcal{L}^{1}$-almost every $s \in A\left(x, v_{i}\right)$. Considering $W_{i}$ as a vector space and using the Fubini's theorem, we get the existence of the limit in (40) for $\mathcal{L}^{n}$-almost every $(x, s) \in W_{i} \times \mathbb{R}$ for which $x \delta_{s} v_{i} \in A$, where $n$ denotes the topological dimension of $\mathbb{G}$. 
Since both $\mathcal{H}^{Q}$ with respect to the homogeneous distance $d$ and $\mathcal{L}^{n}$ with respect to the understood Euclidean metric on $\mathbb{G}$ are Haar measures of $\mathbb{G}$, applying the previous argument to all directions $v_{i}$, for $\mathcal{H}^{Q}$-almost all $x \in A$ all the limits

$$
\partial_{v_{i}} f(x)=\lim _{\substack{t \rightarrow 0 \\ t \in A\left(x, v_{i}\right)}} \delta_{\frac{1}{t}}\left(f(x)^{-1} f\left(x \delta_{t} v_{i}\right)\right)
$$

with $i=1,2, \ldots, N$ exist. Let us fix any $\epsilon>0$. Since $A$ is bounded, in view of both Lusin and Severini-Egorov theorems, we have a compact set $C \subset A$, made of density points, such that for all $i=1,2, \ldots, N$ the limits $\partial_{v_{i}} f(x)$ exist at every point $x \in C$, the convergence

$$
\delta_{\frac{1}{t}}\left(f(x)^{-1} f\left(x \delta_{t} v_{i}\right)\right) \rightarrow \partial_{v_{i}} f(x) \quad \text { as } \quad t \rightarrow 0 \quad \text { and } \quad t \in A\left(x, v_{i}\right)
$$

is uniform on $C$, the maps $x \mapsto \partial_{v_{i}} f(x)$ are continuous on $C$, the convergence of the densities

$$
\frac{\mathcal{H}^{1}\left(B(x, r) \cap A \cap\left\{x \delta_{t} v_{i}: t \in \mathbb{R}\right\}\right)}{2 r} \rightarrow 1
$$

as $r \downarrow 0$ is uniform for $x \in C$ and $\mathcal{H}^{Q}(A \backslash C)<\epsilon$. Let us choose $x \in C$. We know that

$$
\lim _{d(0, z) \rightarrow 0} \frac{d(C, x z)}{d(0, z)}=0 .
$$

Now, we choose $u=\delta_{t_{1}} v_{1} \cdots \delta_{t_{N}} v_{N}$ with $\left|t_{i}\right|<T$ and $t \in(-1,1)$. Thus, we are able to find a sequence $v_{1}^{t}, \ldots, v_{N}^{t} \in \mathbb{G}$ so that

$$
x v_{1}^{t} \cdots v_{i}^{t} \in C \quad \text { and } \quad d\left(v_{i}^{t}, \delta_{t t_{i}} v_{i}\right)=d\left(C, x v_{1}^{t} \cdots v_{i-1}^{t} \delta_{t t_{i}} v_{i}\right)
$$

for all $i=1, \ldots, N$. Such a sequence exists since $C$ is compact. We will also use the elements $w_{1}^{t}, \ldots, w_{N}^{t} \in \mathbb{G}$ such that for all $i=1, \ldots, N$ we have $x v_{1}^{t} \cdots v_{i-1}^{t} w_{i}^{t} \in A$,

$$
w_{i}^{t} \in\left\{\delta_{h} v_{i}: h \in \mathbb{R}\right\} \quad \text { and } \quad d\left(w_{i}^{t}, \delta_{t t_{i}} v_{i}\right)=d\left(\left\{x v_{1}^{t} \cdots v_{i-1}^{t} \delta_{h} v_{i}: h \in \mathbb{R}\right\} \cap A, x v_{1}^{t} \cdots v_{i-1}^{t} \delta_{t t_{i}} v_{i}\right) \text {. }
$$

Such a sequence exists because $A$ is closed and (43) uniformly holds. Moreover, the same uniform convergence of (43) yields

$$
\frac{d\left(w_{i}^{t}, \delta_{t t_{i}} v_{i}\right)}{t} \rightarrow 0
$$

uniformly with respect to $x$ that varies in $C,\left|t_{i}\right|<T$ and $i=1, \ldots, N$, as $t \rightarrow 0$. Notice that we have not emphasized the dependence on $x$. The different sets of projected elements $\left\{v_{i}^{t}\right\}$ and $\left\{w_{i}^{t}\right\}$ are illustrated in Figure 1. For all $t \in(-1,1)$, we have

$$
\delta_{\frac{1}{t}}\left(f(x)^{-1} f\left(x v_{1}^{t} \cdots v_{N}^{t}\right)\right)=\prod_{i=1}^{N} \delta_{\frac{1}{t}}\left(f\left(x v_{1}^{t} \cdots v_{i-1}^{t}\right)^{-1} f\left(x v_{1}^{t} \cdots v_{i}^{t}\right)\right) .
$$

From (43) it follows in particular that whenever $x \in C$ for every $a>0$ the following homogeneity property

$$
\delta_{a} \partial_{v_{i}} f(x)=\partial_{a v_{i}} f(x) .
$$

Thus, we define $\eta_{i}^{t}=x v_{1}^{t} \cdots v_{i-1}^{t} \in C$ for every $i=1, \ldots, N$ and consider the inequality

$$
\begin{aligned}
& \rho\left(\delta_{\frac{1}{t}}\left(f\left(\eta_{i}^{t}\right)^{-1} f\left(\eta_{i}^{t} w_{i}^{t}\right)\right), \partial_{\delta_{t_{i} v_{i}}} f(x)\right) \leq \rho\left(\delta_{\frac{1}{t}}\left(f\left(\eta_{i}^{t}\right)^{-1} f\left(\eta_{i}^{t} w_{i}^{t}\right)\right), \partial_{\delta_{\frac{1}{t}} w_{i}^{t}} f\left(\eta_{i}^{t}\right)\right) \\
& +\rho\left(\partial_{\delta_{\frac{1}{t}} w_{i}^{t}} f\left(\eta_{i}^{t}\right), \partial_{\delta_{t_{i} v_{i}}} f\left(\eta_{i}^{t}\right)\right)+\rho\left(\partial_{\delta_{t_{i} v_{i}}} f\left(\eta_{i}^{t}\right), \partial_{\delta_{t_{i}} v_{i}} f(x)\right) .
\end{aligned}
$$


PSfrag replacements

$x \delta_{t t_{1}} v_{1}$
$x v_{1}^{t}$
$x \delta_{t t_{1}} v_{1} \delta_{t t_{2}} v_{2}$
$x v_{1}^{t} v_{2}^{t}$
$x v_{1}^{t} w_{2}^{t}$
$x$
$A$
$C$

Figure 1. The choice of elements $v_{i}^{t}$ and $w_{i}^{t}$ in the proof of Theorem 1.1.

The first addend on the right hand side uniformly converges to 0 when $x$ varies in $C$, as $t \rightarrow 0$. In fact, $\eta_{i}^{t} \in C$ and the uniform convergence of (41) is preserved under rescaling, where $\delta_{\frac{1}{t}} w_{i}^{t}=\delta_{h_{t} t_{i}} v_{i}$ and $h_{t}$ uniformly converges to one on $C$ as $t \rightarrow 0$, due to (46).

Since we have the rescaling of partial derivatives, we get

$$
\rho\left(\partial_{\delta_{\frac{1}{t}} w_{i}^{t}} f\left(\eta_{i}^{t}\right), \partial_{\delta_{t_{i} v_{i}}} f\left(\eta_{i}^{t}\right)\right)=\rho\left(\delta_{h_{t} t_{i}} \partial_{v_{i}} f\left(\eta_{i}^{t}\right), \delta_{t_{i}} \partial_{v_{i}} f\left(\eta_{i}^{t}\right)\right)
$$

that uniformly goes to zero as $x \in C$ and $t \rightarrow 0$, again due to (46). This implies the uniform convergence of the second addend to zero. Finally, the third term converges uniformly to 0 by the continuity of $\partial_{v_{i}} f$ on $C$. Joining (44) and (45), we have that

$$
\delta_{1 / t}\left(v_{1}^{t} \cdots v_{i}^{t}\right) \rightarrow \prod_{l=1}^{i} \delta_{t_{l}} v_{l}
$$

uniformly with respect to all $i=1, \ldots, N$ and $\left|t_{i}\right|<T$ as $t \rightarrow 0$. It follows that

$$
t^{-1} d\left(v_{i}^{t}, \delta_{t t_{i}} v_{i}\right) \rightarrow 0 \quad \text { as } \quad t \rightarrow 0
$$

for all $i=1, \ldots, N$ and $\left|t_{i}\right|<T$. This convergence is not uniform with respect to $x$, although we could make it even uniform with respect to $x$ by choosing this element in a "slightly smaller subset of $C^{\prime}$. We have $t^{-1} d\left(w_{i}^{t}, v_{i}^{t}\right) \leq t^{-1} d\left(w_{i}^{t}, \delta_{t t_{i}} v_{i}\right)+t^{-1} d\left(\delta_{t t_{i}} v_{i}, v_{i}^{t}\right) \rightarrow 0$ as $t \rightarrow 0$. This gives

$$
\delta_{\frac{1}{t}}\left(f\left(\eta_{i}^{t} w_{i}^{t}\right)^{-1} f\left(\eta_{i}^{t} v_{i}^{t}\right)\right) \rightarrow 0 \quad \text { as } \quad t \rightarrow 0 .
$$

Combining the previous limit with (42), we get that

$$
\delta_{\frac{1}{t}}\left(f\left(\eta_{i}^{t}\right)^{-1} f\left(\eta_{i}^{t} v_{i}^{t}\right)\right)=\delta_{\frac{1}{t}}\left(f\left(\eta_{i}^{t}\right)^{-1} f\left(\eta_{i}^{t} w_{i}^{t}\right)\right) \delta_{\frac{1}{t}}\left(f\left(\eta_{i}^{t} w_{i}^{t}\right)^{-1} f\left(\eta_{i}^{t} v_{i}^{t}\right)\right) \rightarrow \partial_{\delta_{t_{i}} v_{i}} f(x)
$$

as $t \rightarrow 0$. This limit is uniform with respect to all $\left|t_{i}\right|<T$, but it may depend on $x$. Therefore, we are led to the existence of the following limit

$$
\lim _{t \rightarrow 0} \delta_{\frac{1}{t}}\left(f(x)^{-1} f\left(x v_{1}^{t} \cdots v_{N}^{t}\right)\right)=\prod_{i=1}^{N} \partial_{\delta_{t_{i}} v_{i}} f(x),
$$


that is uniform with respect to $u \in V$. This allows us to define $L_{x}(u)=\prod_{i=1}^{N} \partial_{\delta_{t_{i} v_{i}}} f(x)$ for all $u=\delta_{t_{1}} v_{1} \cdots \delta_{t_{N}} v_{N} \in \mathbb{G}$. Therefore the definition of $L_{x}(u)$ is independent of the choice of the representation of $u$. The choice of the "nonlinear difference quotient"

$$
\delta_{\frac{1}{t}}\left(f(x)^{-1} f\left(x v_{1}^{t} \cdots v_{N}^{t}\right)\right)
$$

has been made in order to get the existence of the h-homomorphism $L_{x}$. In fact, $L_{x}$ is an h-homomorphism since for all $u=\delta_{t_{1}} v_{1} \cdots \delta_{t_{N}} v_{N}$ and $w=\delta_{\tau_{1}} v_{1} \cdots \delta_{\tau_{N}} v_{N}$, we have

$$
L_{x}(u w)=\prod_{i=1}^{N} \partial_{\delta_{t_{i} v_{i}}} f(x) \prod_{i=1}^{N} \partial_{\delta_{\tau_{i} v_{i}}} f(x)=L_{x}(u) L_{x}(w) .
$$

The homogeneity of $L_{x}$ is obvious due to the homogeneity of partial derivatives (47). Let $\left(y_{i}\right)_{i=1}^{\infty} \subset A$ be any sequence so that $y_{i} \rightarrow x$. Define $\lambda_{p}=d\left(x, y_{p}\right)$ and let $z_{p} \in \mathbb{G}$ be such that $x^{-1} y_{p}=\delta_{\lambda_{p}} z_{p}$. It follows that

$$
\begin{aligned}
\lim _{p \rightarrow \infty} \frac{\rho\left(f(x)^{-1} f\left(y_{p}\right), L_{x}\left(x^{-1} y_{p}\right)\right)}{d\left(x, y_{p}\right)} & =\lim _{p \rightarrow \infty} \frac{1}{\lambda_{p}} \rho\left(f(x)^{-1} f\left(x \delta_{\lambda_{p}} z_{p}\right), L_{x}\left(\delta_{\lambda_{p}} z_{p}\right)\right) \\
& =\lim _{p \rightarrow \infty} \rho\left(\delta_{\frac{1}{\lambda_{p}}}\left(f(x)^{-1} f\left(x \delta_{\lambda_{p}} z_{p}\right)\right), L_{x}\left(z_{p}\right)\right)=0
\end{aligned}
$$

since both the limits (49) and (48) for $i=N$ are uniform. Thus, $L_{x}$ is the h-differential of $f$ at $x$. The arbitrary choice of $\varepsilon>0$ concludes the proof.

\section{THE METRIC AREA FORMULA}

Let us fix a metric space $Y$ equipped with a distance $\rho$ and let $\mathbb{G}$ denote a stratified group equipped with both a homogeneous distance $d$ and the Hausdorff measure $\mathcal{H}_{d}^{Q}$ constructed with respect to $d$. The integer $Q$ is the homogeneous dimension of $\mathbb{G}$. Since $\mathbb{G}$ is a locally compact real Lie group, the measure $\mathcal{H}_{d}^{Q}$ is the Haar measure of $\mathbb{G}$. For all homogeneous distances $\sigma$ on $\mathbb{G}$, we set $\mathcal{H}_{\sigma}^{Q}=\beta_{Q} h_{\sigma}^{Q}$ and

$$
h_{\sigma}^{Q}(A)=\sup _{\varepsilon>0} \inf \left\{\sum_{j=0}^{\infty} \frac{\operatorname{diam}_{\sigma}\left(E_{j}\right)^{Q}}{2^{Q}}: A \subset \bigcup_{j=0}^{\infty} E_{j}, \operatorname{diam}_{\sigma}\left(E_{j}\right) \leq \varepsilon\right\}
$$

where the constant $\beta_{Q}>0$ is fixed.

Definition 4.1 (Metric differentiability). Let $A \subset \mathbb{G}$ and let $f: A \longrightarrow Y$. We fix a density point $x \in A$. Then we say that $f$ is metrically differentiable at $x$ if there exists a homogeneous seminorm $s$ such that $\rho(f(x), f(x z))-s(z)=o(\|z\|)$ as $z \in x^{-1} A$ and $\|z\| \rightarrow 0^{+}$. The homogeneous seminorm $s$ is unique and it is denoted by $m d f(x)$, which we call the metric differential of $f$ at $x$.

Notice that in the case $\mathbb{G}$ is a Euclidean space, formula (2) yields the Kirchheim's area formula established in [13], where the following notion of Jacobian is used

$$
\mathcal{J}(s)=\frac{n \omega_{n}}{\int_{\mathbb{S}^{n-1}} s(x)^{-n} d \mathcal{H}_{|\cdot|}^{n-1}(x)} .
$$

Here $s$ denotes a seminorm on $\mathbb{R}^{n}$ and $|\cdot|$ is the standard Euclidean norm. In fact, the metric differential in this case is precisely a seminorm. By the special geometric properties 
of normed spaces, Lemma 6 of [13] shows that $\mathcal{J}(\|\cdot\|)=\mathcal{H}_{\|\cdot\|}^{n}(A) / \mathcal{H}_{|\cdot|}^{n}(A)$ for any $A \subset X$ of positive measure, where $X$ is a finite dimensional Banach space with norm $\|\cdot\|$. This immediately shows that formula (3) gives the Kirchheim's Jacobian when the Carnot group is replaced by a Euclidean space. The following remark shows that the metric Jacobian in stratified groups coincides with the sub-Riemannian Jacobian of [16].

Remark 4.1. Let $L: \mathbb{G} \longrightarrow \mathbb{M}$ be an injective h-homomorphism from a stratified group $\mathbb{G}$ to a Banach homogeneous group $\mathbb{M}$. Let us define $s_{L}(x)=\rho(L(x), 0)$ for all $x \in \mathbb{G}$ and notice that $s_{L}$ is a homogeneous distance on $\mathbb{G}$, due to the injectivity of $L$. One can easily check that $\mathcal{H}_{s_{L}}^{Q}\left(B_{1}\right)=\mathcal{H}_{\rho}^{Q}\left(L\left(B_{1}\right)\right)$. It follows that

$$
J\left(s_{L}\right)=\frac{\mathcal{H}_{\rho}^{Q}\left(L\left(B_{1}\right)\right)}{\mathcal{H}_{d}^{Q}\left(B_{1}\right)} .
$$

When $\mathbb{M}$ is in particular a Carnot group, then (51) shows that the metric Jacobian (3) coincides with the "sub-Riemannian Jacobian" introduced in Definition 10 of [16]. One can relate these Jacobians with the classical ones computed by matrices. In fact, as proved in Proposition 3.18 of [16], there is a geometric constant $C$, a priori also depending on the subspace $L(\mathbb{G})$, such that

$$
J\left(s_{L}\right)=C \sqrt{\operatorname{det}\left(L_{0}^{T} L_{0}\right)},
$$

where $L_{0}$ is the matrix representing $L$ as a linear mapping from $\mathbb{G}$ to $L(\mathbb{G})$ with respect to a fixed scalar product. Of course, when one chooses distances with many symmetries the constant $C$ will not depend on the subspace $L(\mathbb{G})$. Formula (52) extends to our framework, since in the general case where the target is a Banach homogeneous group $\mathbb{M}$, we have that $L(\mathbb{G})$ is still a finite dimensional linear subspace of $\mathbb{M}$.

The key ingredient for the area formula is the linearization procedure associated to the a.e. differentiability, see Lemma 3.2.2 of [7]. For metric valued Lipschitz mappings with Euclidean source space, Kirchheim uses the separability of all compact convex sets, according to 2.10.21 of [7], in order to get the following separability of norms: there exists a countable family of norms $\left\{\|\cdot\|_{i}\right\}$ such that for every $0<\varepsilon<1$ and every norm $\|\cdot\|$ we have some $\|\cdot\|_{i_{0}}$ such that $(1-\varepsilon)\|\cdot\|_{i_{0}} \leq\|\cdot\| \leq(1+\varepsilon)\|\cdot\|_{i_{0}}$.

Let us point out that a metric ball with respect to a homogeneous distance need not be a convex set. However, the previous separability of norms still holds for homogeneous norms, since the point is that a Carnot group $\mathbb{G}$ is a boundedly compact metric space and the class of nonempty compact sets in $\mathbb{G}$ is separable with respect to the Hausdorff distance between compact sets.

Lemma 4.1. There exists a countable family $\mathcal{F}$ of homogeneous norms such that for every $\varepsilon \in(0,1)$ and every homogeneous norm $v$, there exists $s \in \mathcal{F}$ such that

$$
(1-\varepsilon) s \leq v \leq(1+\varepsilon) s .
$$

Next, we recall the measure theoretic notion of Jacobian, see [18]. 
Definition 4.2 (Measure theoretic Jacobian). Let $E \subset \mathbb{G}$ be a closed set, $f: E \longrightarrow Y$ Lipschitz and $x \in E$. Then we define

$$
J_{f}(x)=\limsup _{r \rightarrow 0^{+}} \frac{\mathcal{H}_{\rho}^{Q}\left(f\left(E \cap D_{x, r}\right)\right)}{\mathcal{H}_{d}^{Q}\left(D_{x, r}\right)} .
$$

Lemma 4.2. Let $E \subset \mathbb{G}$ be closed and let $f: E \rightarrow Y$ be Lipschitz. Denote by $\mathcal{D} \subset E$ the subset of points where $f$ is metrically differentiable and the metric differential is a homogeneous norm. Then the following statements hold.

(1) There exists a family of Borel sets $\left\{E_{i}\right\}_{i \in \mathbb{N}}$ such that $\mathcal{D}=\bigcup_{i=0}^{\infty} E_{i}$ and $f_{\mid E_{i}}$ is biLipschitz onto its image.

(2) For $\mathcal{H}^{Q}$-a.e. $x \in \mathcal{D}$ we have $J(\operatorname{md} f(x))=J_{f}(x)$.

Proof. Let $\mathcal{F}=\left\{s_{i}: i \in \mathbb{N}\right\}$ be as in Lemma 4.1. Fix an arbitrary $\varepsilon \in(0,1)$ and define for all $i, n \in \mathbb{N}$ the set

$$
\mathcal{D}_{i, n}=\left\{x \in \mathcal{D}:\left|\rho(f(x u), f(x))-s_{i}(u)\right| \leq \varepsilon s_{i}(u) \text { for all } u \in x^{-1} E \text { with }\|u\|<e^{-n}\right\} .
$$

Combining metric differentiability with Lemma 4.1 gives $\mathcal{D}=\bigcup_{i, n \in \mathbb{N}} \mathcal{D}_{i, n}$. Since $\mathbb{G}$ is separable, we can cover $\mathcal{D}_{i, n}$ with a countable family of sets $D_{z l, e^{-n} / 4} \cap \mathcal{D}_{i, n}$ with $l \in \mathbb{N}$. Then for all $x, y \in D_{z_{l}, e^{-n} / 4} \cap \mathcal{D}_{i, n}$, we immediately get

$$
(1-\varepsilon) s_{i}\left(x^{-1} y\right) \leq \rho(f(x), f(y)) \leq(1+\varepsilon) s_{i}\left(x^{-1} y\right) .
$$

This concludes the proof of the first statement. Now, we fix $z \in \mathcal{D}_{i, n} \cap D\left(\mathcal{D}_{i, n}\right)$. Then for all $r \in] 0, e^{-n} / 2$ [ and $x, y \in D_{z, r} \cap \mathcal{D}_{i, n}$, the inequalities (54) again hold. As a consequence, setting $L=\operatorname{Lip}(f)$, we get

$$
\frac{\mathcal{H}_{\rho}^{Q}\left(f\left(D_{z, r} \cap E\right)\right)}{\mathcal{H}_{d}^{Q}\left(D_{z, r}\right)} \leq(1+\varepsilon)^{Q} \frac{\mathcal{H}_{s_{i}}^{Q}\left(D_{z, r}\right)}{\mathcal{H}_{d}^{Q}\left(D_{z, r}\right)}+L^{Q} \frac{\mathcal{H}_{d}^{Q}\left(D_{z, r} \backslash \mathcal{D}_{i, n}\right)}{\mathcal{H}_{d}^{Q}\left(D_{z, r}\right)}
$$

and

$$
\frac{\mathcal{H}_{\rho}^{Q}\left(f\left(D_{z, r} \cap E\right)\right)}{\mathcal{H}_{d}^{Q}\left(D_{z, r}\right)} \geq \frac{\mathcal{H}_{\rho}^{Q}\left(f\left(D_{z, r} \cap \mathcal{D}_{i, n}\right)\right)}{\mathcal{H}_{d}^{Q}\left(D_{z, r}\right)} \geq(1-\varepsilon)^{Q} \frac{\mathcal{H}_{s_{i}}^{Q}\left(D_{z, r} \cap \mathcal{D}_{i, n}\right)}{\mathcal{H}_{d}^{Q}\left(D_{z, r}\right)} .
$$

Therefore we have

$$
(1-\varepsilon)^{Q} \frac{\mathcal{H}_{s_{i}}^{Q}\left(D_{1}\right)}{\mathcal{H}_{d}^{Q}\left(D_{1}\right)} \leq \limsup _{r \rightarrow 0^{+}} \frac{\mathcal{H}_{\rho}^{Q}\left(f\left(D_{z, r} \cap E\right)\right)}{\mathcal{H}_{d}^{Q}\left(D_{z, r}\right)} \leq(1+\varepsilon)^{Q} \frac{\mathcal{H}_{s_{i}}^{Q}\left(D_{1}\right)}{\mathcal{H}_{d}^{Q}\left(D_{1}\right)},
$$

for an arbitrary $\varepsilon>0$. This leads us to the conclusion.

Lemma 4.3. Let $f: \mathbb{G} \longrightarrow Y$ be a Lipschitz mapping. Let $E_{0}$ be the set of points $x \in \mathbb{G}$ for which there exists $v_{x} \in \mathbb{G}$ with $\left\|v_{x}\right\|=1$ such that for all $\varepsilon>0$ we have $0<r_{\varepsilon}<\varepsilon$ such that

$$
\rho\left(f\left(x \delta_{r} v_{x}\right), f(x)\right) \leq \varepsilon r_{\varepsilon}
$$

for all $0<r \leq r_{\varepsilon}$. It follows that $\mathcal{H}_{\rho}^{Q}\left(f\left(E_{0}\right)\right)=0$. 
Proof. Let $L \geq 1$ denote a Lipschitz constant of $f$, choose $x \in E_{0}$ and fix an arbitrary $R>0$. Take an arbitrary $\varepsilon>0$ such that $4 \varepsilon<1$ and $B_{x, \varepsilon} \subset B_{R}$. By definition of $E_{0}$, we have $v_{x} \in \mathbb{S}$ and $0<r_{\varepsilon}<\varepsilon / 2$ such that (55) holds. Defining

$$
S_{v_{x}, \varepsilon}=\bigcup_{0 \leq r \leq r_{\varepsilon}} B_{x \delta_{r} v_{x}, \frac{\varepsilon r_{\varepsilon}}{L}} \subset B_{x, \varepsilon},
$$

by triangle inequality, we get $f\left(S_{v_{x}, \varepsilon}\right) \subset B_{\rho}\left(f(x), 2 \varepsilon r_{\varepsilon}\right)$. Now, fix $N=[L / 2 \varepsilon]$ and choose two distinct integers $i, j$ between 0 and $N$. By triangle inequality, one observes that

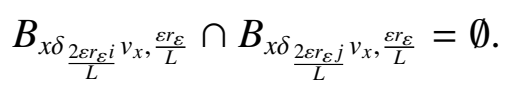

This leads us to $\mathcal{H}_{d}^{Q}\left(S_{v_{x}, \varepsilon}\right)>C \varepsilon^{Q-1} r_{\varepsilon}^{Q}$. As a result, the measure $\mu_{R}=\left(f_{\mid B_{R}}\right)_{\sharp} \mathcal{H}_{\rho}^{Q}$ on $Y$ satisfies

$$
\frac{\mu_{R}\left(B_{\rho}\left(f(x), 2 \varepsilon r_{\varepsilon}\right)\right)}{\left(2 \varepsilon r_{\varepsilon}\right)^{Q}} \geq \frac{C}{2^{Q_{\varepsilon}}} .
$$

From the arbitrary choice of $\varepsilon>0$, it follows that

$$
\limsup _{t \rightarrow 0^{+}} \frac{\mu_{R}\left(B_{\rho}(f(x), t)\right)}{t^{Q}}=+\infty
$$

for every $x \in E_{0} \cap B_{R}$, where $\mu_{R}$ is a finite Borel regular measure on $Y$. Finally, standard differentiation theorems give $\mathcal{H}_{\rho}^{Q}\left(f\left(E_{0} \cap B_{R}\right)\right)=0$ and the arbitrary choice of $R>0$ concludes the proof.

Corollary 4.1. Let $E \subset \mathbb{G}$ be a closed set and let $f: E \longrightarrow Y$ be a Lipschitz mapping, whose metric differential exists on a subset $E_{0} \subset E$ and at all point of this set it is not a homogeneous norm. Then $\mathcal{H}_{\rho}^{Q}\left(f\left(E_{0}\right)\right)=0$.

Proof. The image $f(E)$ is separable in $Y$, so in particular it is a separable metric space that can be isometrically embedded into $l^{\infty}$. Hence we can assume that $Y=l^{\infty}$. With this target, the componentwise extension of $f$ immediately yields a Lipschitz extension $\tilde{f}$ defined on all of $\mathbb{G}$, having the same Lipschitz constant. Take $x \in E_{0} \subset D(E)$ and $v_{x} \in \mathbb{G}$ such that $\left\|v_{x}\right\|=1$ and $m d f(x)\left(v_{x}\right)=0$. It is easy to check that $\tilde{f}$ is metrically differentiable at $x$ and $m d f(x)=m d \tilde{f}(x)$. Therefore $\tilde{f}$ satisfies conditions of Lemma 4.3 and our claim holds.

Proof of Theorem 1.2. Since $\mathcal{H}_{d}^{Q}$ is Borel regular and $f$ is Lipschitz, it is not restrictive to assume that $A$ is closed and that $f$ is everywhere metrically differentiable. By definition of metric Jacobian, $J(m d f(z))=0$ whenever $z$ belongs to the subset $A_{0}$ of $A$ where the metric differential is not a homogeneous norm. Corollary 4.1 implies $\mathcal{H}_{\rho}^{Q}\left(f\left(A_{0}\right)\right)=0$, hence (2) holds for the restriction $f_{\mid A_{0}}$. By Lemma 4.2, we have $A \backslash A_{0}=\bigcup_{j=0}^{\infty} E_{j}$ where $E_{j}$ are Borel sets and $f_{\mid E_{j}}$ is injective and $J_{f}$ equals $J(m d f(\cdot)) \mathcal{H}^{Q}$-almost everywhere, hence Theorem 2 of [18] establishes (2) for $f_{\mid\left(A \backslash A_{0}\right)}$ and concludes the proof.

\section{REFERENCES}

[1] Y. Benyamini, J. Lindenstrauss, Geometric nonlinear functional analysis. Vol. 1, American Mathematical Society Colloquium Publications, Vol. 48, Providence: American Mathematical Society, 2000

[2] J. CHeEger, B. KLEINER, Differentiability of Lipschitz maps from metric measure spaces to Banach spaces with the Radon-Nikodým property, Geom. Funct. Anal. 19, n.4, 1017-1028, (2009) 
[3] J. Cheeger, B. Kleiner, "Differentiating maps into $L^{1}$ and the geometry of BV functions", Ann. of Math. (2) 171, no. 2 (2010): 1347-1385

[4] J. Cheeger, B. Kleiner, "Metric differentiation, monotonicity and maps to $L^{1}$ ", Invent. Math. 182, no. 2 (2010): 335-70

[5] E. B. Dynkin, "Computation of the coefficients in the formula of Campbell-Hausdorff", (Russian), Dokl. Akad. Nauk. SSSR (N.S.) 57 , (1947): 323-26

[6] E. B. Dynkin, "Normed Lie Algebras and Analytic Groups", Amer. Math. Soc. Transl., no. 97 (1953): 470-535

[7] H. Federer, Geometric Measure Theory, New York: Springer-Verlag New York Inc., 1969

[8] G. B. Folland, E. M. Stein, Hardy Spaces on Homogeneous groups, Princeton: Princeton University Press, 1982

[9] R. W. Goodman, Nilpotent Lie groups: structure and applications to analysis, Lecture Notes in Mathematics, 562, Berlin-New York: Springer-Verlag, 1976

[10] M. Gromov, "Carnot-Carathéodory spaces seen from within". In Subriemannian Geometry, Progress in Mathematics, 144, edited by A. Bellaiche and J. Risler, 79-323. Basel: Birkhäuser Verlag, 1996

[11] W. B. Johnson, J. Lindenstrauss, G. Schechtman, "Extensions of Lipschitz maps into Banach spaces", Israel Journal of Math., 54, no. 2 (1986): 129-38

[12] M. Karmanova, S. Vodop'yanov, “Geometry of Carnot-Carathéodory spaces, differentiability, coarea and area formulas". In Analysis and mathematical physics, Trends Math, 233-335. Basel: Birkhäuser, 2009

[13] B. KirchHeIm, "Rectifiable metric spaces: local structure and regularity of the Hausdorff measure", Proc. Amer. Math. Soc. 121 (1994): 113-23

[14] J. R. LeE, A. NAOR. " $L_{p}$ metrics on the Heisenberg group and the Goemans-Linial conjecture", In Proceedings of 47th Annual IEEE Symposium on Foundations of Computer Science (FOCS 2006), IEEE Computer Society, (2006): 99-108

[15] B. Kirchieim, V. Magnani, "A counterexample to metric differentiability”, Proc. Ed. Math. Soc. 46 (2003): 221-227

[16] V. Magnani, "Differentiability and Area formula on stratified Lie groups", Houston J. Math., 27, no. 2 (2001): 297-323

[17] V. Magnani, "Unrectifiability and rigidity in stratified groups", Arch. Math. 83, no. 6 (2004): 568-76

[18] V. Magnani, "An area formula in metric spaces", Coll. Math. 124, no.2 (2011): 275-83

[19] P. Pansu, "Métriques de Carnot-Carathéodory quasiisométries des espaces symétriques de rang un", Ann. Math. 129 (1989): 1-60

[20] S. D. PAuls, “The Large Scale Geometry of Nilpotent Lie groups”, Comm. Anal. Geom. 9, no. 5 (2001): 951-982

[21] K. Rogovin, "Non-smooth analysis in infinite dimensional Banach homogenous groups", J. Convex Anal. 14, no. 4 (2007): 667-91

[22] A.D.Ukhlov, S.K.Vodop' Yanov, "Approximately differentiable transformations and change of variables on nilpotent groups", Siberian Math. J. 37, no. 1 (1996): 62-78

[23] S.SEmmes, On the non existence of bilipschitz parametrization and geometric problems about $A_{\infty}$ weights, Rev. Mat. Iberoamericana, 12, 337-410, (1996).

[24] E. M. SteIn, Harmonic Analysis: Real-Variable Methods, Orthogonality, and Oscillatory Integrals, Princeton: Princeton University Press, 1993

Valentino Magnani, Dipartimento di Matematica, Largo Bruno Pontecorvo 5, 56127, Pisa, Italy

E-mail address: magnani@dm.unipi.it

Tapio Rajala, Scuola Normale Superiore, Piazza dei Cavalieri 7, 56127, Pisa, Italy

E-mail address: tapio.rajala@sns.it 